\title{
Operations in the homology spectral sequence of a cosimplicial infinite loop space
}

\author{
Philip Hackney ${ }^{1}$ \\ Department of Mathematics, University of California, Riverside, 900 University Avenue, \\ Riverside, CA 92521, USA
}

\begin{abstract}
Consider the mod 2 homology spectral sequence associated to a cosimplicial space $X$. We construct external operations whose target is the spectral sequence associated to $E \Sigma_{2} \times_{\Sigma_{2}}(X \times X)$. If $X$ is a cosimplicial $E_{\infty}$-space, we couple these external operations with the structure map

$$
E \Sigma_{2} \times \Sigma_{2}(X \times X) \rightarrow X
$$

to produce internal operations in the spectral sequence. In the sequel we show that they agree with the usual Araki-Kudo operations on the abutment $H_{*}(\operatorname{Tot} X)$.
\end{abstract}

Keywords: Araki-Kudo operation, cosimplicial space, spectral sequence 2000 MSC: 55S12, 55T20

\section{Introduction}

Let $\mathcal{C}$ be a fixed $E_{\infty}$ operad in spaces. The aim of this work is to provide a new proof of the following theorem.

Theorem 1.1. Suppose that $X$ is a cosimplicial object in the category of $\mathcal{C}$ spaces. Then there are operations in the mod-2 homology spectral sequence associated to $X$ :

$$
\begin{array}{lll}
Q^{m}: E_{-s, t}^{r} \rightarrow E_{-s, m+t}^{r} & & m \geq t \\
Q^{m}: E_{-s, t}^{r} \rightarrow E_{m-s-t, 2 t}^{w} & & m \in[t-s, t]
\end{array}
$$

where $w \in[r, 2 r-2]$ is given by

$$
w= \begin{cases}r & m=t-s \\ 2 r-2 & m \in[t-s+1, t-r+2] \\ r+t-m & m \in[t-r+3, t] .\end{cases}
$$

Email address: hackney@math.ucr.edu (Philip Hackney)

${ }^{1}$ Phone: 951-827-5402, Fax: 951-827-7314

Preprint submitted to Elsevier

July 26, 2021 
The first proof of this was given by Jim Turner in [22]. We present here a fundamentally different proof which is more direct and is amenable to generalization. In particular, in [9], we prove the analogue of Theorem 1.1 for cosimplicial $E_{n+1}$-spaces. The calculations for the $E_{\infty}$-case are substantially more transparent than those for the $E_{n+1}$ case, and [9] requires much of the machinery and many of the calculations in this paper. Had we bundled this into one paper, we would have had to prove the $E_{\infty}$ case first anyway, so for simplicity we have separated it out. Another motivation for providing a new proof of 1.1 is to address the issue of compatibility with Araki-Kudo operation\& 2 in the abutment of the spectral sequence, $H_{*}(\operatorname{Tot} X)$. Thus this paper also feeds into [10], where some of the themes (small examples and external operations) help us to give the first complete proof of [22, 5.11] (the proof sketch given there has substantial gaps).

Pictorially, the images of these operations applied to an element in bidegree $(-s, t)$ lie on the solid and dotted lines of Figure 1. We call operations of the first type "vertical operations" and operations of the second type "horizontal operations".

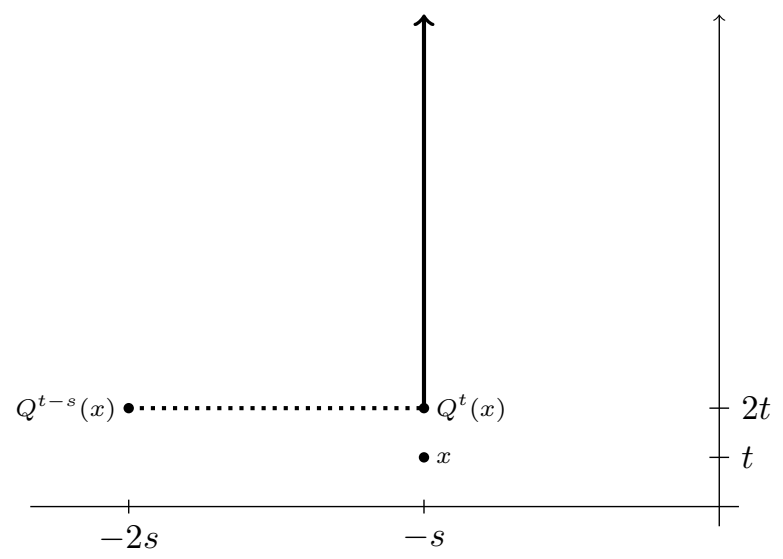

Figure 1: Vertical and Horizontal Operations

Prior to Turner's work, Araki-Kudo operations were constructed in EilenbergMoore spectral sequences in [1] and [14], though in both cases only the 'vertical' operations were given. The theory of Steenrod operations, on the other hand, has a rich literature. Appropriate starting points are [7] and [21, Chapter 7]. Steenrod operations in spectral sequences have been used to facilitate computations of cohomology; in particular, this extra structure sometimes allows one to prove collapse theorems. As an example, the existence of Steenrod operations was used in [18] to calculate the cohomology of the classifying spaces of some exceptional Lie groups.

\footnotetext{
${ }^{2}$ These are also referred to as Dyer-Lashof operations.
} 
We now mention two examples of cosimplicial $\mathcal{C}$-spaces where the homology spectral sequence and its target are of interest. The theory presented in this sequence of papers may provide insight to each. For the first, consider a pullback diagram

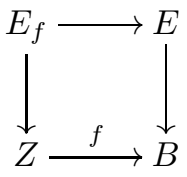

and let $X^{\bullet}$ be the two-sided geometric cobar construction with $X^{n}=Z \times B^{n} \times E$ (see [19, §2] for a complete definition). Using $H_{*}$ to denote homology mod 2 , the homology spectral sequence associated to $X^{\bullet}$ is the Eilenberg-Moore spectral sequence

$$
E^{2} \cong \operatorname{Cotor}^{H_{*}(B)}\left(H_{*}(E), H_{*}(Z)\right) \Rightarrow H_{*}\left(E_{f}\right),
$$

as demonstrated in [19, Theorem 5.1]. If the above pullback diagram is a pullback diagram in the category of $\mathcal{C}$-spaces, then $X^{\bullet}$ is a cosimplicial $\mathcal{C}$-space. Thus, in this case, there are operations in the Eilenberg-Moore spectral sequence associated to the pullback diagram above.

As a second example, suppose that $Z$ is a space and that $R$ is a commutative ring. In [5, Chapter I], Bousfield and Kan define the $R$-completion of $Z$ as the totalization of a certain cosimplicial space $R Z$. It turns out that if $Z$ is a $\mathcal{C}$ space, then $\underset{\sim}{R Z}$ is a cosimplicial $\mathcal{C}$-space. Thus there are homology operations in the associated spectral sequence, which, coupled with convergence results of Bousfield and Shipley [4, 20], may help provide information about the mod 2 homology of $R$-completions of various $\mathcal{C}$-spaces.

The scheme of the paper is as follows. We work with simple Bousfield-Kan universal examples, which one might think of as cosimplicial spheres. We construct external operations for these examples and use the universal property to transport these operations into the spectral sequence for an arbitrary cosimplicial space. When that cosimplicial space is actually a cosimplicial $\mathcal{C}$-space, we then obtain internal operations by combining the external operations with the $\mathcal{C}(2)$-structure.

We begin with some background and notation.

\subsection{Homology Spectral Sequence and Passage to Chains}

Let $X$ be a cosimplicial space (where 'space' means either topological space or simplicial set). We briefly outline the construction of the homology spectral sequence associated to $X$ (see [4] for more details).

Definition. We always work over the field $\mathbb{k}=\mathbb{Z} / 2$ and just write $\mathrm{Ch}$ for the category of chain complexes over $\mathbb{k}$. For a set $Z$ with basepoint $*$, the notation $\mathbb{k} Z$ will always mean the free $\mathbb{k}$-module with basis $Z \backslash\{*\}$, so that $\mathbb{k}$ defines a functor $\operatorname{Set}_{*} \rightarrow \mathbb{k}$ Mod. If $Z$ is just a set, then $\mathbb{k} Z$ will be denote the free $\mathbb{k}$-module with basis $Z$. 
We will use the normalization functor $N: \mathbb{k} \operatorname{Mod}^{\Delta^{o p}} \rightarrow$ Ch given, on a simplicial $\mathbb{k}$-module $Z$, by

$$
N_{k}(Z)=Z_{k} /\left(s_{0} Z_{k-1}+\ldots s_{k-1} Z_{k-1}\right)
$$

with differential induced from the sum of the face maps. Let

$$
S_{*}: \text { Spaces } \rightarrow \mathrm{Ch}
$$

be the mod-2 (normalized) chains functor. The first step in the construction of the spectral sequence is to pass from $X$ to the cosimplicial chain complex $S_{*}(X)$.

If $Y$ is a cosimplicial object in an abelian category $\mathcal{A}$, we will write $C Y$ for the conormalization of $Y$

$$
C Y^{p}=\operatorname{coker}\left(\bigoplus_{k=1}^{p} d^{k}: \bigoplus Y^{p-1} \rightarrow Y^{p}\right)
$$

which is an object in $\operatorname{coCh}^{\geq 0} \mathcal{A}$, the category of nonnegative cochain complexes over $\mathcal{A}$. The differential on $C Y$, which we write as $d_{h}$, is the map induced by the coface map $d^{0}$. When $\mathcal{A}=\mathrm{Ch}$, the category of chain complexes over $\mathbb{k}$, we will regard $C Y$ as a left-plane bicomplex which consists of the $\mathbb{k}$-module $C Y_{q}^{p}$ in bidegree $(-p, q)$.

Definition. In this setting, we always write

$$
d_{v}: C Y_{q}^{p} \rightarrow C Y_{q-1}^{p}
$$

for the differential coming from $Y^{p}$ and

$$
d_{h}: C Y_{q}^{p} \rightarrow C Y_{q}^{p+1}
$$

for the differential which is induced from the coface maps. Given the definition of conormalization, we have that $d_{h}$ is just the coface map $d^{0}$. We will also write $\partial=d_{v}+d_{h}$ for the differential in the total complex below.

Given a bicomplex $B$, we will let Tot $B$ denote the product total complex:

$$
(\operatorname{Tot} B)_{m}=\prod_{j} B_{j, m-j} .
$$

The appropriate filtration in this situation is the one by columns

$$
F_{m}^{k}=\prod_{j \leq k} B_{j, m-j}
$$

We may regard Tot $C(Y)$ as a subcomplex of

$$
\prod_{m} \operatorname{Hom}\left(\Delta_{*}^{m}, Y^{m}\right)
$$


where this is the internal Hom in the category $\mathrm{Ch}$ and $\Delta_{*}^{m}$ denotes normalized simplicial chains on the simplicial set $\Delta^{m}=\operatorname{Hom}(-,[m])$. The natural filtration of $\Delta_{*}^{\bullet}$ by skeleta induces the above filtration on Tot $C(Y)$ (see [4]).

The homology spectral sequence associated to a cosimplicial space $X$ is, by definition, the one obtained from this filtration on $\operatorname{Tot} C S_{*}(X)$. For this reason we usually work with cosimplicial chain complexes rather than cosimplicial spaces, though of course we will have to check that various geometric constructions we make behave well when we pass to chains. This will usually take the form of an $E^{1}$ or $E^{2}$ isomorphism between algebraic and geometric constructions.

Definition. If $C$ is a filtered chain complex with $F^{-s-1} \subset F^{-s}$, we will write

$$
\begin{aligned}
& Z_{-s, t}^{r}=\left\{x \in F^{-s} C_{t-s} \mid \partial x \in F^{-s-r} C\right\} \\
& B_{-s, t}^{r}=\partial Z_{-s+r-1, t-r+2}^{r-1}+Z_{-s-1, t+1}^{r-1}
\end{aligned}
$$

for the $r$-cycles and $r$-boundaries, and $E_{-s, t}^{r}=Z_{-s, t}^{r} / B_{-s, t}^{r}$ for the $(-s, t)$ position of the $r^{\text {th }}$ page of the spectral sequence. Furthermore, if $x$ and $y$ are in $F^{-s} C_{t-s}$, then we will write $x \sim_{r} y$ if $x-y \in B_{-s, t}^{r}$.

\subsection{External Operations}

Araki-Kudo operations were introduced in [13]. In this section we recall, following the construction in [16], that the operations for a $\mathcal{C}$-space $X$ may be obtained by combining an "external operation" with the $\mathcal{C}(2)$-structure map.

Set $\pi:=\Sigma_{2}=\{e, \sigma\}$ and let $\mathbb{k}^{\text {triv }}$ be $\mathbb{k}$ with trivial $\pi$-action. Let $W$ be the minimal $\mathbb{k} \pi$-free resolution of $\mathbb{k}^{\text {triv }}$, which is defined by

$$
W_{i}= \begin{cases}\mathbb{k} \pi \cdot e_{i} & i \geq 0 \\ 0 & i<0\end{cases}
$$

and

$$
d\left(e_{i}\right)=(1+\sigma) e_{i-1} .
$$

This is $\mathbb{k} \pi$-chain-homotopic to $S_{*}(E \pi)$, which, combined with the shuffle map, gives a quasi-isomorphism

$$
W \otimes_{\pi}\left(S_{*}(X) \otimes S_{*}(X)\right) \rightarrow S_{*}\left(E \pi \times_{\pi}(X \times X)\right)
$$

for any space $X$. We have that $\mathcal{C}(2)$ is equivariantly homotopic to $E \pi$, so if $X$ is a $\mathcal{C}$-space then there is a map

$$
E \pi \times{ }_{\pi}(X \times X) \rightarrow X
$$

which induces

$$
W \otimes_{\pi}\left(S_{*}(X) \otimes S_{*}(X)\right) \rightarrow S_{*}(X)
$$


Let $C$ be a chain complex. We define, for each $m$, a graded (non-additive) function of degree $m$

$$
\begin{aligned}
q^{m}: C & \rightarrow W \otimes_{\pi}(C \otimes C) \\
c & \mapsto e_{m-|c|} \otimes c \otimes c+e_{m+1-|c|} \otimes c \otimes d c
\end{aligned}
$$

(interpreting terms with $e_{-n}$ as zero). Notice that $q^{m} d=d q^{m}$ and that $q^{m}$ induces a homomorphism in homology. If $C$ is a chain complex equipped with a map $W \otimes_{\pi}(C \otimes C) \rightarrow C$ (for example if $C$ is chains on a $\mathcal{C}$-space) then the image of $[c]$ under the composite

$$
H_{*}(C) \rightarrow H_{*+m}\left(W \otimes_{\pi}(C \otimes C)\right) \rightarrow H_{*+m}(C)
$$

is, by definition, $Q^{m}[c]$. Since the Araki-Kudo operations $Q^{m}$ factor through $H_{*}\left(W \otimes_{\pi}(C \otimes C)\right)$, we call

$$
q^{m}: H_{*}(C) \rightarrow H_{*+m}\left(W \otimes_{\pi}(C \otimes C)\right)
$$

an 'external operation'.

A naive first attempt to construct operations in our spectral sequence would involve mimicking what we did for chain complexes. We now explore this, though it won't be enough, as noted below. The tensor product of two cosimplicial chain complexes $A_{*}^{\bullet}$ and $B_{*}^{\bullet}$ is the cosimplicial chain complex given in cosimplicial degree $p$ by $A_{*}^{p} \otimes B_{*}^{p}$. If $X$ is a cosimplicial $\mathcal{C}$-space, then we have a map

$$
W \otimes_{\pi}\left(S_{*}(X) \otimes S_{*}(X)\right) \stackrel{E^{1} \text {-iso. }}{\longrightarrow} S_{*}\left(E \pi \times_{\pi}(X \times X)\right) \rightarrow S_{*} X,
$$

so it is useful to consider cosimplicial chain complexes $Y$ equipped with a map

$$
W \otimes_{\pi}(Y \otimes Y) \rightarrow Y .
$$

If $Y$ is any cosimplicial chain complex, then applying $q^{m}$ levelwise gives a map of cosimplicial sets

$$
q^{m}: Y \rightarrow W \otimes_{\pi}(Y \otimes Y) .
$$

One must then check (since $q^{m}$ is not additive) that this induces maps $E^{r}(Y) \rightarrow$ $E^{r}\left(W \otimes_{\pi}(Y \otimes Y)\right)$ for $r \geq 1$. Combining this with (4) gives operations

$$
Q^{m}: E^{r}(Y) \stackrel{q^{m}}{\rightarrow} E^{r}\left(W \otimes_{\pi}(Y \otimes Y)\right) \rightarrow E^{r}(Y) .
$$

Notice, though, that $[y] \in E_{-s, t}^{r}$ is mapped to something in bidegree $(-s, t+m)$ and to zero for $m<t$, so we only pick up the vertical part of Figure 1.

Henceforth, whenever we speak of external operations on a cosimplicial chain complex $Y$ we will mean operations whose target is the spectral sequence for $W \otimes_{\pi}(Y \otimes Y)$. It will grow quite tedious to write

$$
W \otimes_{\pi}(Y \otimes Y)
$$

for the homotopy orbit complex, so instead we will abbreviate it as

$$
\mathcal{E}(Y)=W \otimes_{\pi}(Y \otimes Y) .
$$


Remark (See Section[10). The class $[y]$ is in total degree $t-s$, so we expect there to be Araki-Kudo operations in total degrees $\geq 2(t-s)$. The vertical operations begin in total degree $2 t-s$, indicating that we have missed a few. There is one other operation that we could reasonably talk about here, the one at the bottom left. Notice that if $Y$ comes equipped with a map $W \otimes_{\pi}(Y \otimes Y) \rightarrow Y$, then there is a multiplication on the spectral sequence of $Y$. Since the bottom Araki-Kudo operation of an element is meant to be its square, it is compelling to notice that if $[y]$ is in $E_{-s, t}^{r}$ then both $Q^{t-s}[y]$ and $[y]^{2}$ are in bidegree $(-2 s, 2 t)$. This may convince the skeptical reader of the validity of the shape of Figure 1 .

\subsection{Bousfield-Kan Universal Examples}

For each $p$, define $\mathbf{D}_{(r, s, s)}^{p}$ as the cofiber of the inclusion

$$
\mathrm{sk}_{s-1} \Delta_{+}^{p} \rightarrow \mathrm{sk}_{s+r-1} \Delta_{+}^{p}
$$

in the category of simplicial based sets (here $\Delta_{+}^{p}$ is obtained by adding a disjoint basepoint to the standard simplicial $p$-simplex). For $t \geq s$, define $\mathbf{D}_{(r, s, t)}^{p}$ by iterating the Kan suspension $([12,2.2]$ or $[8$, III.5]) $t-s$ times.

$$
\mathbf{D}_{(r, s, t)}^{p}=\Sigma^{t-s} \mathbf{D}_{(r, s, s)}^{p}
$$

These cosimplicial spaces $\mathbf{D}_{(r, s, t)}^{\bullet}$ were introduced in [6] where it was shown that the (integral) homology spectral sequence has the form of Figure 2 ,

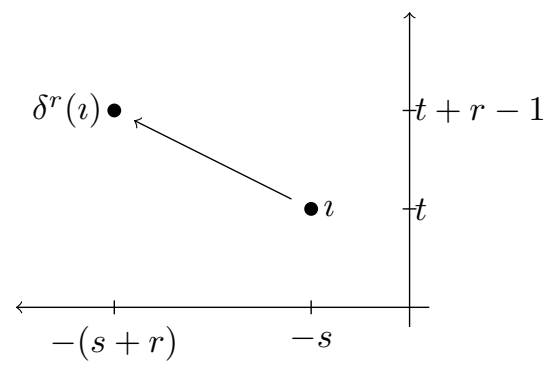

Figure 2: Spectral Sequence for $\mathbf{D}_{(r, s, t)}$

The Bousfield-Kan example $\mathbf{D}_{(r, s, t)}$ is weakly universal for elements in $E_{-s, t}^{r}$ of the homology spectral sequence. Indeed, for a cosimplicial simplicial abelian group $B$ and an element $b \in E_{-s, t}^{r}(B)$ there is a map of cosimplicial simplicial abelian groups $\mathbb{Z} \mathbf{D}_{(r, s, t)} \rightarrow B$ which, on the spectral sequence level, sends $\imath$ to $b$. We used the adjective 'weakly' because the cosimplicial simplicial abelian group map actually depends on a choice of representative of $b$. Slightly more general ideas can be found in [6], while slightly more specific ideas can be found in section 2.2

In any case, the spaces $\mathbf{D}_{(r, s, t)}$ are the atomic cosimplicial spaces when it comes to the homology spectral sequence. To understand external operations, 


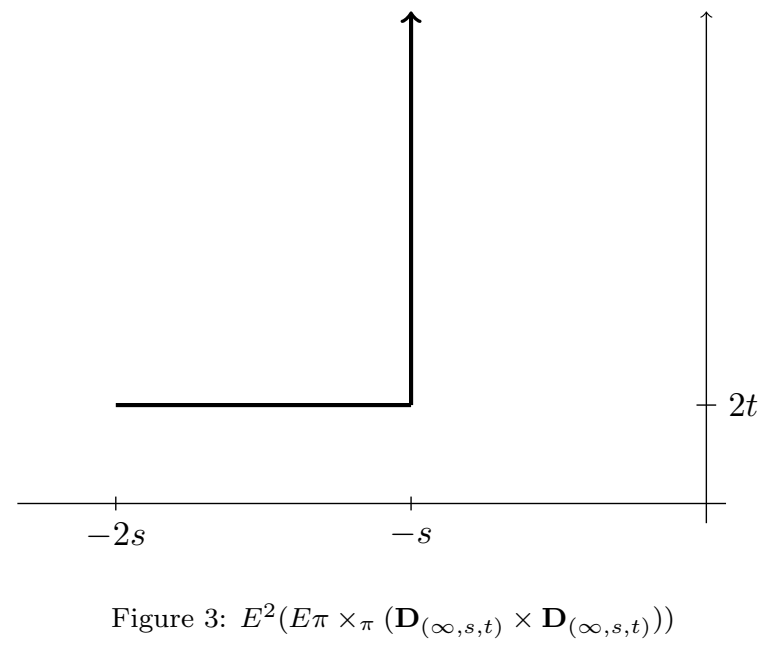

we will first understand them in these basic examples. We shall examine the spectral sequence for the cosimplicial space $E \pi \times_{\pi}\left(\mathbf{D}_{(r, s, t)} \times \mathbf{D}_{(r, s, t)}\right)$.

Part of the proof of Theorem 1.1] we present relies on a calculation (Theorem 7.1) giving Figure 3 (or Figure 8 on page 25), which is extremely suggestive when compared to Figure 1 on page 2

\subsection{Outline}

The next section is devoted to a careful consideration of the universal examples of Bousfield and Kan. In particular, we give a basis for the levelwise homology (Proposition 2.3), a complete calculation of the spectral sequence (Proposition 2.4), and the universal property (Proposition 2.5). The basis for the levelwise homology will be used in Section 3 to give a basis for the $E^{1}$ page of the spectral sequence associated to $\mathcal{E}\left(D_{r s t}\right)$, which is the spectral sequence we spend much of the remainder of the paper calculating. We explore some consequences of the dual Eilenberg-Zilber theorem for cosimplicial chain complexes in Section 4, which we will need for the calculation of $E^{2}$ in Sections 54 Sections 8 and 9 are dedicated to a computation of the higher differentials in this spectral sequence.

Having completed the calculation of the spectral sequence associated to $\mathcal{E}\left(D_{r s t}\right)$, we give a definition in Section 10 of the external operations on the level of cycles. The basic idea is that an $r$-cycle $y \in Z_{-s, t}^{r}(Y)$ determines a map $\Theta_{y}: D_{r s t} \rightarrow Y$ with $\imath \mapsto[y]$. We define the operations on $[y]$ as the images of certain generators of $E^{r}\left(\mathcal{E}\left(D_{r s t}\right)\right)$ (the bottom right part of Figure 8 on page 25) under the map induced by $\mathcal{E}\left(\Theta_{y}\right)$. It is not at all obvious from this construction (perhaps because it is not generally true) that these operations depend only on the class $[y] \in E_{-s, t}^{r}(Y)$, rather than on the $r$-cycle $y$, nor is it obvious that the operations are homomorphisms. In Section 111 we show that these cyclelevel operations are additive, which we use finally in Section 12 to show that the cycle-level operations descend to spectral sequence operations in the sense 
of Theorem 1.1. The fact that the representing maps depend on our choice of $r$-cycle introduces some indeterminacy in this step, which is precisely where the ' $w$ ' in the theorem statement comes in. On page 41 we use the universal examples to show that this ' $w$ ' is the best possible.

\section{Algebraic Bousfield-Kan Examples}

In this section we give an explicit description of the mod-2 homology spectral sequence associated to the Bousfield-Kan universal examples.

We give two separate constructions of the $E^{1}$ page of these spectral sequences. The second, starting on page 12, gives a complete description of the spectral sequence and allows us to establish the universal property for the Bousfield-Kan universal examples. It is also relatively quick.

The first construction is more involved and only produces $E^{1}$. This relies on the observation (see [6, 3.1]) that, for a cosimplicial chain complex $Y$,

$$
C\left(H_{t}(Y)\right)^{s} \cong H_{t}\left(C Y^{s}\right),
$$

where the latter term is isomorphic to $E_{-s, t}^{1}(Y)$. The method we use is to first compute the levelwise homology $H_{t}\left(Y^{s}\right)$ and then calculate the (higher) coface maps $H_{t}\left(d^{1}\right), H_{t}\left(d^{2}\right), \ldots, H_{t}\left(d^{s+1}\right)$. This will tell us about the left-hand side of the above isomorphism.

The advantage to presenting a construction along these lines is two-fold. First, it provides good practice since we will use this method of calculation later to calculate $\mathcal{E}(Y)$. More importantly, the levelwise homology of $Y$ gives information about the levelwise homology of $\mathcal{E}(Y)$ (see section 3.1).

Most future calculations in this paper rely on the bases we choose here.

\subsection{Homology of the Skeleton of the p-Simplex}

Let $\Delta^{p}$ denote the (normalized) simplicial chains for the standard simplicial model of the $p$-simplex. A basis for $\Delta^{p}$ in dimension $k$ is given by the set of ordered injections $[k] \hookrightarrow[p]$.

For a complex $C$, write $\operatorname{sk}_{t}(C)$ for the brutal truncation with

$$
\operatorname{sk}_{t}(C)_{k}= \begin{cases}C_{k} & k \leq t \\ 0 & k>t\end{cases}
$$

This notation is chosen because of its relation to the usual notion of simplicial skeleton: if $X$ is a simplicial set, then

$$
\mathrm{sk}_{t} S_{*} X=S_{*} \mathrm{sk}_{t} X,
$$

where $S_{*}=N \mathbb{k}$ is the normalized simplicial $\mathbb{k}$-chains functor. Thus we wish to compute the homology of $\mathrm{sk}_{t} \Delta^{p}$, and since we always have

$$
H_{k}\left(\operatorname{sk}_{t} C\right)= \begin{cases}0 & k>t \\ Z_{t}(C) & k=t \\ H_{k}(C) & k<t\end{cases}
$$


we are left to understand $Z_{t}\left(\Delta^{p}\right)$. If $t>0$ then $Z_{t}\left(\Delta^{p}\right)=B_{t}\left(\Delta^{p}\right)$ and if $t=0$ then $Z_{t}\left(\Delta^{p}\right)$ is given by the collection of vertices $\mathbb{k}\{[0] \hookrightarrow[p]\}$. In summary,

$$
H_{k}\left(\mathrm{sk}_{t} \Delta^{p}\right)= \begin{cases}B_{t}\left(\Delta^{p}\right) & k=t>0 \\ \mathbb{k}\{[0] \hookrightarrow[p]\} & k=t=0 \\ \mathbb{k} & k=0, t>k \\ 0 & \text { else. }\end{cases}
$$

We now give an explicit description for $H_{t}\left(\mathrm{sk}_{t} \Delta^{p}\right)=B_{t}\left(\Delta^{p}\right)$ when $t>0$.

Definition. We already have $\Delta_{r}^{p}=\mathbb{k}\{[r] \hookrightarrow[p]\}$. Consider the set of based injections

$$
\Lambda_{r}^{p}=\{\varepsilon \mid \varepsilon:[r] \hookrightarrow[p], \varepsilon(0)=0\} .
$$

Proposition 2.1. The restriction of the differential

$$
d_{v}: \Delta_{t+1}^{p} \rightarrow \Delta_{t}^{p}
$$

for $t \geq 0$ gives an isomorphism

$$
\mathbb{k} \Lambda_{t+1}^{p} \stackrel{\cong}{\rightrightarrows} B_{t}\left(\Delta^{p}\right) .
$$

Proof. We define an operator $S: \Delta_{t}^{p} \rightarrow \Delta_{t+1}^{p}$ whose image is $\mathbb{k} \Lambda_{t+1}^{p}$. This operator takes a basis element $\varepsilon:[t] \hookrightarrow[p]$ to zero if $0 \in \operatorname{im} \varepsilon$, or, if $0 \notin \operatorname{im} \varepsilon$, adds zero to its image so that $\varepsilon$ decomposes as

$$
[t] \stackrel{d^{0}}{\rightarrow}[t+1] \stackrel{S(\alpha)}{\longrightarrow}[p] .
$$

Then $S^{2}=0$ and $d_{v} S+S d_{v}=\operatorname{id}_{[t]}$ for $t>0$.

To show surjectivity of $d_{v}: \operatorname{im} S \rightarrow B_{t}\left(\Delta^{p}\right)$, consider a boundary $d_{v} \varepsilon$ (with $\varepsilon:[t+1] \hookrightarrow[p]$ for some $t+1 \geq 1)$. Then

$$
d_{v} \varepsilon=d_{v}\left(d_{v} S \varepsilon+S d_{v} \varepsilon\right)=d_{v}\left(S d_{v} \varepsilon\right)
$$

is in the image of $\left.d_{v}\right|_{\mathrm{im} S}$.

To show injectivity, suppose $S \varepsilon:[t+1] \hookrightarrow[p]$ is in $\operatorname{im} S=\mathbb{k} \Lambda_{t+1}^{p}$. If $d_{v}(S \varepsilon)=0$, then

$$
S \varepsilon=S\left(S d_{v} \varepsilon+d_{v} S \varepsilon\right)=S^{2} d_{v} \varepsilon+S\left(d_{v}(S \varepsilon)\right)=0 .
$$

Proposition 2.2. The homology of the t-skeleton of the standard p-simplex is given by

$$
H_{k}\left(\operatorname{sk}_{t} \Delta^{p}\right) \cong \begin{cases}\mathbb{k} \Lambda_{t+1}^{p} & k=t>0 \\ \mathbb{k}\{[0] \hookrightarrow[p]\} & k=t=0 \\ \mathbb{k} & k=0, t>k \\ 0 & \text { else. }\end{cases}
$$




\subsection{Homology Spectral Sequence of the Bousfield-Kan Examples}

Fix $r \geq 1, s, t \geq 0$, and define $D^{p}=D_{r s s}^{p}$ as the cokernel of the inclusion

$$
\mathrm{sk}_{s-1} \Delta^{p} \hookrightarrow \mathrm{sk}_{s+r-1} \Delta^{p}
$$

The cosimplicial structure of $D^{\bullet}$ is induced from that of $\Delta^{\bullet}$. It is not hard to see, for $t \geq s$, that

$$
D_{r s t}:=\Sigma^{t-s} D_{r s s} \cong N \mathbb{k} \mathbf{D}_{(r, s, t)},
$$

where $\mathbf{D}_{(r, s, t)}$ is the cosimplicial space defined in [6, 5.1] and (6), $N$ is the nor-

malization functor $\mathbb{k} \mathrm{Mod}^{\Delta^{o p}} \rightarrow \mathrm{Ch}$, and $\Sigma$ is the suspension of chain complexes:

$$
(\Sigma C)_{q+1}=C_{q} .
$$

Proposition 2.3. For $s \geq 0$ and $r \geq 2$, the homology of $D_{r s s}^{p}$ is given by

$$
H_{k}\left(D_{r s s}^{p}\right) \cong \begin{cases}\mathbb{k} \Lambda_{s+r}^{p} & k=s+r-1 \\ \mathbb{k} \Lambda_{s}^{p} & k=s \\ 0 & \text { else. }\end{cases}
$$

Proof. There is a short exact sequence of complexes

$$
0 \rightarrow \operatorname{sk}_{s-1} \Delta^{p} \rightarrow \operatorname{sk}_{s+r-1} \Delta^{p} \rightarrow D^{p} \rightarrow 0
$$

and, when $s \neq 1$, the result follows immediately from the associated long exact sequence and Proposition 2.2. When $s=1$, the bottom map in the diagram

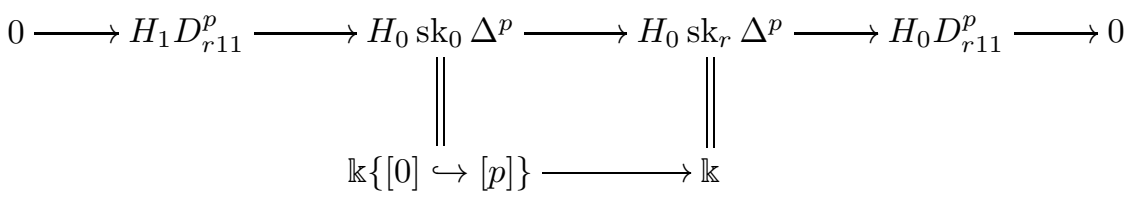

is surjective and $\mathbb{k} \Lambda_{1}^{p}$ is a $p$-dimensional vector space.

Remark. The statement of this proposition is not true for $r<2$. We will assume that $r \geq 2$ until section 11, where we will momentarily need an easy calculation for $r=1$.

We now reproduce a the mod-2 version of [6, 5.3, (i)-(iii)] using Proposition 2.3. Namely, we compute the $E^{1}$ page of the spectral sequence of $D_{r s s}$. We do this using the fact (from [․, 3.1$]$ ) that

$$
E_{-p, q}^{1}\left(D_{r s s}\right)=H_{q} C\left(D_{r s s}\right)^{p} \cong C\left(H_{q}\left(D_{r s s}\right)\right)^{p} .
$$

There isn't an obvious way to obtain information about the differentials from this isomorphism, so we will not prove $[6,5.3$, (iv)] using this method. The answer is given in Figure 4 (see also Figure 6). 


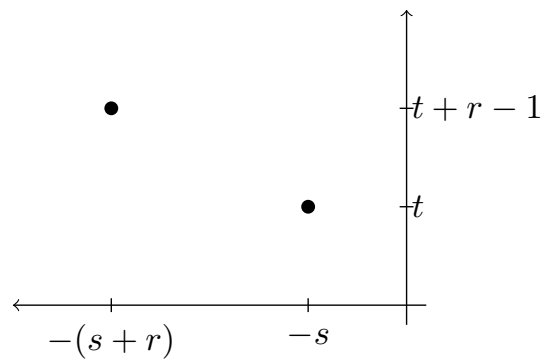

Figure 4: Page 1 of $D_{r s t}$

We want to compute a basis for $C H D_{\text {rss }}$ (noting that $C H D_{\text {rst }}$ is obtained from this by suspension). For $j>0$, the coface map $d^{j}$ takes elements of $\Lambda_{t}^{p}$ to elements of $\Lambda_{t}^{p+1}$. Applying conormalization, we find

$$
\begin{aligned}
C H_{s}\left(D_{r s s}\right)^{p} & =\mathbb{k} \Lambda_{s}^{p} /\left(d^{1} \mathbb{k} \Lambda_{s}^{p-1}+\cdots+d^{p} \mathbb{k} \Lambda_{s}^{p-1}\right) \\
& \cong \mathbb{k}\{\varepsilon \mid \varepsilon:[s] \hookrightarrow[p], \varepsilon(0)=0,[1, p] \subset \operatorname{im} \varepsilon\} \\
& =\mathbb{k}\left\{\operatorname{id}_{[s]}\right\} \\
C H_{s+r-1}\left(D_{r s s}\right)^{p} & \cong \mathbb{k} \Lambda_{s+r}^{p} /\left(d^{1} \mathbb{k} \Lambda_{s+r}^{p-1}+\cdots+d^{p} \mathbb{k} \Lambda_{s+r}^{p-1}\right) \\
& \cong \mathbb{k}\{\varepsilon \mid \varepsilon:[s+r] \hookrightarrow[p], \varepsilon(0)=0,[1, p] \subset \operatorname{im} \varepsilon\} \\
& =\mathbb{k}\left\{\operatorname{id}_{[s+r]}\right\} \\
C H_{k}\left(D_{r s s}\right)^{p} & =0 \quad k \neq s, s+r-1 .
\end{aligned}
$$

This is reflected in Figure 4 The separation of the two remaining classes means all intervening differentials $\delta^{1}, \delta^{2}, \ldots, \delta^{r-1}$ must be zero, so $E^{1}=E^{2}=\cdots=$ $E^{r}$.

Instead of using Proposition 2.3, we could conormalize $D_{r s s}$ and get Figure 5 The elements on the line with $y$-intercept 0 are $\operatorname{id}_{[s]}, \ldots, \operatorname{id}_{[s+r-1]}$ and those on the line with $y$-intercept -1 are $d_{h} \operatorname{id}_{[s]}, \ldots, d_{h} \operatorname{id}_{[s+r-1]}$. One can calculate that

$$
d_{h} \operatorname{id}_{[k]}=d_{v} \operatorname{id}_{[k+1]}
$$

in $C \Delta^{\bullet}$ since $d_{v} \operatorname{id}_{[k+1]}=\operatorname{id}_{[k+1]} d^{0}=d^{0}=d_{h}=d_{h} \operatorname{id}_{[k]}$.

Proposition 2.4. If $2 \leq r \leq \infty$, then

$$
E_{p, q}^{1}\left(D_{r s t}\right)=E_{p, q}^{r}\left(D_{r s t}\right)= \begin{cases}\mathbb{k} & (p, q)=(-s, t) \text { or }(-s-r, t+r-1) \\ 0 & \text { otherwise. }\end{cases}
$$

If, furthermore, $r<\infty$, then $E^{r+1}\left(D_{r s t}\right)=0$. Finally,

$$
H_{k}\left(\operatorname{Tot} C D_{r s t}\right)= \begin{cases}\mathbb{k} & k=t-s \text { and } r=\infty \\ 0 & \text { otherwise }\end{cases}
$$

where Tot is the product totalization (2). 


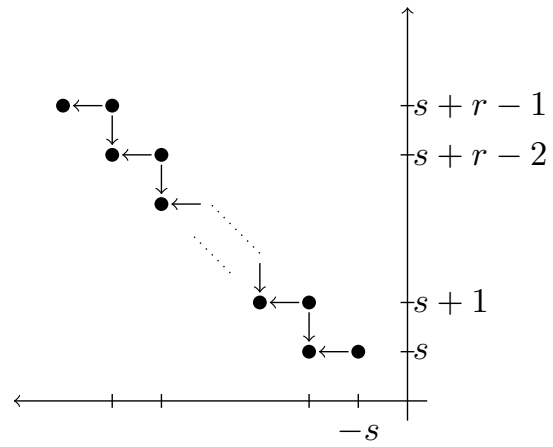

Figure 5: The Bicomplex $C\left(D_{r s s}\right)$

Proof. This essentially follows from Figure 5 in the case $s=t$ and by iterated suspension otherwise. There is exactly one $r$-cycle in total degree 0 , namely

$$
\imath=\sum_{k=s}^{s+r-1} \operatorname{id}_{[k]} .
$$

Applying the total differential, we have $\partial \imath=d_{h} \operatorname{id}_{[s+r-1]}($ or 0 if $r=\infty)$.

This proof tells us why $E^{r+1} D_{r s s}=0$ for $r<\infty$, which we did not show using the first construction. Thus, the spectral sequence is as in Figure 6 .

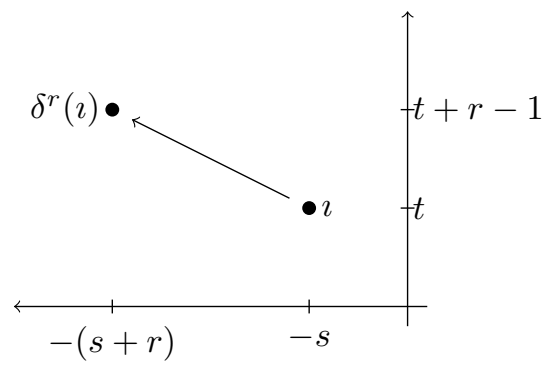

Figure 6: Pages 2 through $r$ of $D_{r s t}$

Proposition 2.5 (Universal Property). Let $Y$ be a cosimplicial chain complex and $y \in Z_{-s, t}^{r}(Y)$. Then there is a map

$$
\Theta_{y}: D_{r s t} \rightarrow Y
$$

with

$$
E^{r}\left(\Theta_{y}\right)(\imath)=[y] \quad E^{r}\left(\Theta_{y}\right)\left(d_{h} \operatorname{id}_{[s+r-1]}\right)=\delta^{r}[y] .
$$

We will be using the definition of $\Theta_{y}$ frequently. 
Definition (Representing Map). Let

$$
y \in Z_{-s, t}^{r}(Y) \subset F^{-s} \operatorname{Tot} C(Y)_{t-s}=\prod_{p \geq s} C Y_{t-s+p}^{p}
$$

which we write as

$$
y=\sum_{k=0}^{\infty} y_{t+k}^{s+k} \quad y_{q}^{p} \in C(Y)_{q}^{p}
$$

Define $C\left(\Theta_{y}\right)$ by

$$
\begin{aligned}
\Sigma^{t-s} \operatorname{id}_{[s+k]} & \mapsto y_{t+k}^{s+k} \\
\Sigma^{t-s} d_{h} \operatorname{id}_{[s+k]} & \mapsto d_{h} y_{t+k}^{s+k} .
\end{aligned}
$$

The following proof will show that this is a map of bicomplexes, so $C\left(\Theta_{y}\right)$ gives $\Theta_{y}$ by the Dold-Kan theorem.

Proof of Proposition 2.5. Since $\partial y \in F^{-s-r}$ we know that

$$
d_{h} y_{t+k}^{s+k}=d_{v} y_{t+k+1}^{s+k+1}
$$

for $0 \leq k \leq r-2$, which shows that $C\left(\Theta_{y}\right)$ is a map of bicomplexes. Furthermore, using the notation from page 5 , we have

$$
C\left(\Theta_{y}\right) \imath=\sum_{k=0}^{r-1} y_{t+k}^{s+k} \sim_{r} \sum_{k=0}^{\infty} y_{t+k}^{s+k}=y
$$

and

$$
C\left(\Theta_{y}\right) \partial \imath=d_{h} y_{t+r-1}^{s+r-1}
$$

\section{The $E^{1}$ Page}

We are interested in the spectral sequence associated to the cosimplicial chain complex $\mathcal{E}\left(D_{r s t}\right)$ (where $\mathcal{E}$ is a homotopy orbit complex, see (5)). Note that $\mathcal{E}\left(D_{r s t}\right) \cong \Sigma^{2 t-2 s} \mathcal{E}\left(D_{r s s}\right)$ where the suspension is taken levelwise, so it is enough to understand the spectral sequence for $\mathcal{E}\left(D_{\text {rss }}\right)$. Let $Y$ be a cosimplicial chain complex; following [6, 3.1], $E_{-p}^{1}(Y)$ is isomorphic to $C\left(H_{*} Y\right)^{p}$. We note that the isomorphism $C H_{*}(-) \cong H_{*} C(-)$ is an isomorphism of complexes of graded modules. It is not true in general that the spectral sequence associated to $Y$ is isomorphic to the spectral sequence associated to $H_{*}(Y)$.

Fix $r$ and $s$; we now turn to computing $C\left(H_{*} \mathcal{E}\left(D_{r s s}\right)\right)$. Below we often use the abbreviation $D^{p}=D_{r s s}^{p}$. 


\subsection{Homology}

To compute $H_{*}\left(\mathcal{E}\left(D_{\text {rss }}\right)\right)$, first notice that, for a map of chain complexes $C \rightarrow C^{\prime}$ and choices of quasi-isomorphisms $H(C) \rightarrow C$ and $H\left(C^{\prime}\right) \rightarrow C^{\prime}$, the diagram

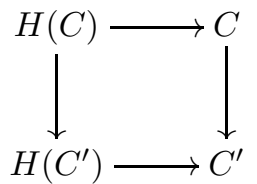

commutes up to homotopy. It follows from [16, Lemma 1.1] that we have a natural transformation

$$
H_{*}\left(\mathcal{E}\left(H_{*}(-)\right)\right) \rightarrow H_{*}(\mathcal{E}(-))
$$

which is an isomorphism for each object. Thus

$$
H_{*}\left(\mathcal{E}\left(D_{r s s}^{p}\right)\right) \cong H_{*}\left(\mathcal{E}\left(H_{*}\left(D_{r s s}^{p}\right)\right)\right)
$$

and this is an isomorphism of cosimplicial graded modules.

Once we have made this change, notice that, for a $\mathbb{k} \pi$-module $M$ (such as $\left.H_{*}\left(D^{p}\right) \otimes H_{*}\left(D^{p}\right)\right)$, the complex $W \otimes_{\pi} M$ is just

$$
\cdots M \stackrel{1+\sigma}{\longrightarrow} M \stackrel{1+\sigma}{\longrightarrow} M \stackrel{1+\sigma}{\longrightarrow} M \rightarrow 0 .
$$

Thus the homology is $M /(1+\sigma)$ in the bottom dimension and $\operatorname{ker}(1+\sigma) / \mathrm{im}(1+$ $\sigma)$ in dimensions bigger than zero. This essentially gives the following special case of [16, Lemma 1.3]:

Lemma 3.1. Let $K$ be a $\mathbb{k}$-module with totally ordered basis $\left\{x_{j} \mid j \in J\right\}$. Let $A \subset K \otimes K$ have basis $\left\{x_{j} \otimes x_{j} \mid j \in J\right\}$ and $B \subset K \otimes K$ have basis $\left\{x_{j_{1}} \otimes x_{j_{2}} \mid j_{1}<j_{2}\right.$, where $\left.j_{1}, j_{2} \in J\right\}$. Then

$$
H\left(W \otimes_{\pi}(K \otimes K)\right) \cong\left(\bigoplus_{i=0}^{\infty} e_{i} \otimes A\right) \oplus\left(e_{0} \otimes B\right) .
$$

We choose an order for the basis $\Lambda_{s}^{p} \sqcup \Lambda_{s+r}^{p}$ of $H_{*}\left(D_{r s s}^{p}\right)$ that we found in Proposition 2.3 .

Definition (Total Order). We associate to $\varepsilon:[m] \hookrightarrow[p]$ the word of length $p+1$, whose $i^{\text {th }}$ letter is 0 if $i \notin \operatorname{im} \varepsilon$ and 1 if $i \in \operatorname{im} \varepsilon$. For a fixed $m$ we declare the order on injections to be given by the reverse lexicographic order on their associated words. By Proposition 2.3 we then have an induced order on $H_{s+r-1}\left(D^{p}\right)$ and on $H_{s}\left(D^{p}\right)$. We give $H_{*}\left(D^{p}\right)$ an order by declaring that $H_{s}\left(D^{p}\right)<H_{s+r-1}\left(D^{p}\right)$. 
We apply Lemma 3.1 with this totally ordered basis to see that the homology of $\mathcal{E}\left(H_{*}\left(D^{p}\right)\right)$ has a basis given by the disjoint union of the following sets:

$$
\begin{gathered}
\left\{e_{m} \otimes \varepsilon \otimes \varepsilon \mid \varepsilon \in \Lambda_{s}^{p}, m \in \mathbb{N}\right\} \\
\left\{e_{m} \otimes \gamma \otimes \gamma \mid \gamma \in \Lambda_{s+r}^{p}, m \in \mathbb{N}\right\} \\
\left\{e_{0} \otimes \varepsilon \otimes \varepsilon^{\prime} \mid \varepsilon, \varepsilon^{\prime} \in \Lambda_{s}^{p}, \varepsilon<\varepsilon^{\prime}\right\} \\
\left\{e_{0} \otimes \varepsilon \otimes \gamma \mid \varepsilon \in \Lambda_{s}^{p}, \gamma \in \Lambda_{s+r}^{p}\right\} \\
\left\{e_{0} \otimes \gamma \otimes \gamma^{\prime} \mid \gamma, \gamma^{\prime} \in \Lambda_{s+r}^{p}, \gamma<\gamma^{\prime}\right\}
\end{gathered}
$$

Each $\gamma$ appearing in the above tensor products stands for $d_{v} \gamma$ as in the isomorphism in Proposition 2.3. This isomorphism is induced from $d_{v}$ (see Proposition 2.1) and, for $k>0, d^{k} d_{v} \gamma=d_{v} d^{k} \gamma$. Thus we may use $d^{k}: \mathbb{k} \Lambda_{s+r}^{p} \rightarrow \mathbb{k} \Lambda_{s+r}^{p+1}$ for $k>0$ without worry.

Lemma 3.2. For $k>0$, the coface maps

$$
d^{k}: \mathcal{E}\left(H_{*}\left(D^{p}\right)\right) \rightarrow \mathcal{E}\left(H_{*}\left(D^{p+1}\right)\right)
$$

respect the basis for $H_{*}\left(\mathcal{E}\left(H_{*}\left(D^{\bullet}\right)\right)\right)$ given above (9)).

Proof. In the word associated to an injection, the operator $d^{k}$ inserts a 0 in the $k^{\text {th }}$ position, an operation which preserves the reverse lexicographic order.

\subsection{Conormalization}

We just saw that $d^{k}, k>0$, sends basis elements in $H_{*}\left(\mathcal{E}\left(H_{*}\left(D^{p}\right)\right)\right)$ to basis elements in $H_{*}\left(\mathcal{E}\left(H_{*}\left(D^{p+1}\right)\right)\right)$ via

$$
\begin{gathered}
e_{m} \otimes \varepsilon \otimes \varepsilon^{\prime} \mapsto e_{m} \otimes d^{k} \varepsilon \otimes d^{k} \varepsilon^{\prime} \\
\varepsilon, \varepsilon^{\prime} \in \Lambda_{s}^{p} \cup \Lambda_{s+r}^{p} \quad m \geq 0 .
\end{gathered}
$$

Thus the conormalization (see (1))

$$
C H_{*}\left(\mathcal{E}\left(H_{*}\left(D^{p}\right)\right)\right)
$$

has a basis consisting of elements of the original basis which are not in the image of $d^{k}$ for $k=1, \ldots, p$.

Theorem 3.3. Let $2 \leq r \leq \infty$ and $s \geq 0$. The $E^{1}$ page of the spectral sequence for the cosimplicial chain complex $\mathcal{E}\left(D_{r s t}\right)$ can be given a basis consisting of the following:

If $r=\infty$, then the basis consists of the disjoint union of the following two sets:

$$
\begin{aligned}
& \left\{e_{m} \otimes \operatorname{id}_{[s]} \otimes \operatorname{id}_{[s]} \in E_{-s, 2 t+m}^{1} \mid m \geq 0\right\} \\
& \left\{e_{0} \otimes \varepsilon \otimes \varepsilon^{\prime} \in E_{-p, 2 t}^{1} \mid \varepsilon<\varepsilon^{\prime} \in \Lambda_{s}^{p} \text { and }[p]=\operatorname{im} \varepsilon \cup \operatorname{im} \varepsilon^{\prime}\right\} .
\end{aligned}
$$


If $r<\infty$, then the basis is given by the disjoint union of the sets (10) with the sets

$$
\begin{aligned}
& \left\{e_{m} \otimes \operatorname{id}_{[s+r]} \otimes \operatorname{id}_{[s+r]} \in E_{-s-r, 2 t+2 r+m-2}^{1} \mid m \geq 0\right\} \\
& \left\{e_{0} \otimes \varepsilon \otimes \gamma \in E_{-p, 2 t+r-1}^{1} \mid \varepsilon \in \Lambda_{s}^{p}, \gamma \in \Lambda_{s+r}^{p} \text { and }[p]=\operatorname{im} \varepsilon \cup \operatorname{im} \gamma\right\} \\
& \left\{e_{0} \otimes \gamma \otimes \gamma^{\prime} \in E_{-p, 2 t+2 r-2}^{1} \mid \gamma<\gamma^{\prime} \in \Lambda_{s+r}^{p} \text { and }[p]=\operatorname{im} \gamma \cup \operatorname{im} \gamma^{\prime}\right\} .
\end{aligned}
$$

Proof. In the spectral sequence associated to $\mathcal{E}\left(D_{r s s}\right), E_{-p}^{1} \cong C H_{*}\left(\mathcal{E}\left(H_{*}\left(D^{p}\right)\right)\right)$ has a basis consisting of elements of the original basis which are not in the image of $d^{k}$ for $k=1, \ldots, p$. This basis is given by the disjoint union of the following sets:

$$
\begin{gathered}
\left\{e_{m} \otimes \varepsilon \otimes \varepsilon \mid \varepsilon \in \Lambda_{s}^{p},[1, p] \subset \operatorname{im} \varepsilon, m \in \mathbb{N}\right\} \\
\left\{e_{m} \otimes \gamma \otimes \gamma \mid \gamma \in \Lambda_{s+r}^{p},[1, p] \subset \operatorname{im} \gamma, m \in \mathbb{N}\right\} \\
\left\{e_{0} \otimes \varepsilon \otimes \varepsilon^{\prime} \mid \varepsilon, \varepsilon^{\prime} \in \Lambda_{s}^{p},[1, p] \subset \operatorname{im} \varepsilon \cup \operatorname{im} \varepsilon^{\prime}, \varepsilon<\varepsilon^{\prime}\right\} \\
\left\{e_{0} \otimes \varepsilon \otimes \gamma \mid \varepsilon \in \Lambda_{s}^{p}, \gamma \in \Lambda_{s+r}^{p},[1, p] \subset \operatorname{im} \varepsilon \cup \operatorname{im} \gamma\right\} \\
\left\{e_{0} \otimes \gamma \otimes \gamma^{\prime} \mid \gamma, \gamma^{\prime} \in \Lambda_{s+r}^{p},[1, p] \subset \operatorname{im} \gamma \cup \operatorname{im} \gamma^{\prime}, \gamma<\gamma^{\prime}\right\} .
\end{gathered}
$$

Furthermore, recall that $\varepsilon$ and $\gamma$ are injective maps and 0 is in the image of each. This establishes the $s=t$ case of the theorem. Now apply the $(2 t-2 s)$-fold suspension in the vertical direction to this basis.

A picture of the $E^{1}$ page is given in Figure 7 , where we have indicated modules with rank greater than zero by snaky lines and modules of rank one with straight lines. The reader is encouraged to compare this to the picture of $E^{2}$ given in Figure 8 on page 25

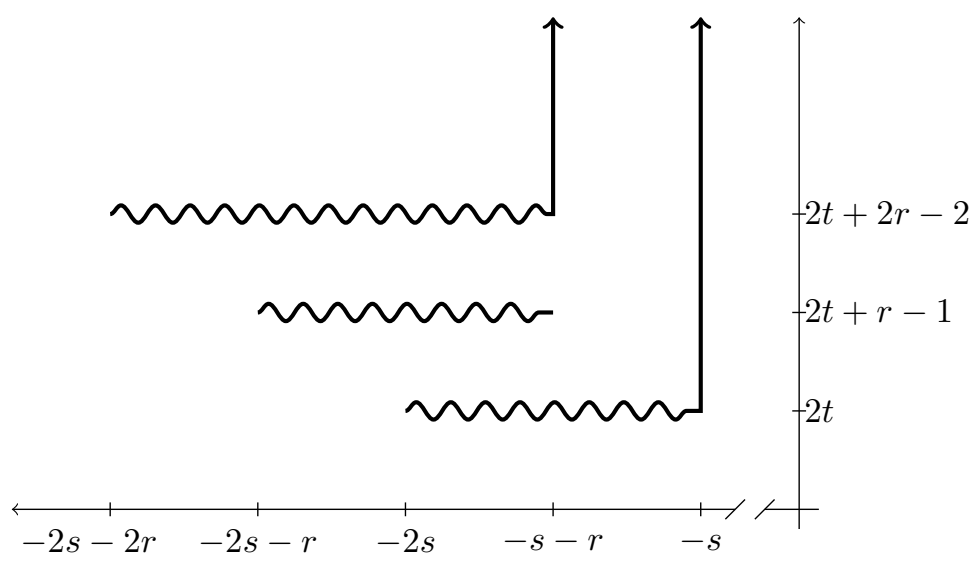

Figure 7: $E^{1}\left(\mathcal{E}\left(D_{r s t}\right)\right)$

Remark. Using the notation of Theorem 3.3. we note that that the basis elements $e_{0} \otimes \varepsilon \otimes \varepsilon^{\prime}$ live in cosimplicial degrees between $-s-1$ and $-2 s, e_{0} \otimes \varepsilon \otimes \gamma$ 
live in cosimplicial degrees between $-s-r$ and $-2 s-r$, and $e_{0} \otimes \gamma \otimes \gamma^{\prime}$ live in cosimplicial degrees between $-s-r-1$ and $-2 s-2 r$.

It is possible to give an explicit description of the differential $\delta^{1}$ in terms of this basis. The interested reader may find formulas in [11], but they are not needed for the calculation of $E^{2}$.

\section{Spectral Sequence of $X \otimes Y$}

In this section we examine the spectral sequence associated to the tensor product of two cosimplicial chain complexes, anticipating applications for computing $E^{2}\left(\mathcal{E}\left(D_{r s t}\right)\right)$ (in section 5) and for the external product (in section 10).

Let $X$ and $Y$ be cosimplicial chain complexes. There are two bicomplexes, $C(X) \otimes C(Y)$ and $C(X \otimes Y)$, which are readily associated to the pair. We now give natural transformations

$$
C(X) \otimes C(Y) \rightleftarrows C(X \otimes Y),
$$

which are the cosimplicial versions of the Alexander-Whitney and shuffle maps on normalized chains that arise when considering simplicial abelian groups, as in [15, Corollaries VIII.8.6 and VIII.8.9].

Definition ([2, Appendix]). The Alexander-Whitney map $A W$ is defined on $C(X)^{p} \otimes C(Y)^{q}$ by

$$
A W\left(x^{p} \otimes y^{q}\right)=d^{p+q} \cdots d^{p+1} x \otimes d^{p-1} \cdots d^{0} y .
$$

The shuffle map $\nabla$ is defined on $C(X \otimes Y)^{n}$ by

$$
\nabla\left(x^{n} \otimes y^{n}\right)=\sum_{p+q=n} \sum_{(p, q)-\text { shuffles }} s^{\tau(p)} \cdots s^{\tau(p+q-1)} x \otimes s^{\tau(0)} \cdots s^{\tau(p-1)} y
$$

where we consider $(p, q)$-shuffles as permutations of the set

$$
\{0,1, \ldots, p+q-1\} .
$$

Lemma 4.1. The Alexander-Whitney map and shuffle map are maps of bicomplexes.

Proof. Regarding $X$ and $Y$ simply as cosimplicial modules, the usual statement is that these are both maps in $\mathrm{Ch}^{\geq 0}(\mathbb{k}$ Mod). Since the cosimplicial operators commute with the internal differential $X_{i}^{p} \rightarrow X_{i-1}^{p}$, these are actually both maps in $\mathrm{Ch}^{\geq 0}(\mathrm{Ch})$. Finally, then tensor product on $\mathrm{Ch}^{\geq 0}(\mathrm{Ch})$ is given by

$$
(A \otimes B)_{q}^{p}=\oplus_{i+j=p} A^{i} \otimes B^{j}=\oplus_{i+j=p, m+n=q} A_{m}^{i} \otimes B_{n}^{j}
$$

which is compatible with the isomorphism between the categories of bicomplexes and cochain complexes in complexes. 
Notice that $C(X) \otimes C(Y)$ is a retraction of $C(X \otimes Y)$ :

$$
\nabla \circ A W=\operatorname{id}_{C(X) \otimes C(Y)} .
$$

Furthermore, if $X$ and $Y$ are cosimplicial abelian groups, the dual EilenbergZilber theorem (see, for example, the appendix in 2]) tells us that $\nabla$ and $A W$ are inverse chain homotopy equivalences. In the case when $X$ and $Y$ are cosimplicial chain complexes, we can extend this to show that these maps give isomorphisms on $E^{2}$ (Proposition 4.3).

We begin with a small result on the spectral sequence associated to the tensor product of bicomplexes.

Lemma 4.2. Let $B$ and $B^{\prime}$ be bicomplexes (over $\mathbb{k}$ ) and consider the spectral sequences obtained by filtering by columns. Then the map

$$
E^{r}(B) \otimes E^{r}\left(B^{\prime}\right) \rightarrow E^{r}\left(B \otimes B^{\prime}\right)
$$

is an isomorphism.

We will generally identify these two bigraded modules.

Proof of Lemma 4.2. The tensor product

$$
\left(B \otimes B^{\prime}\right)_{p, q}=\bigoplus_{i, j} B_{i, j} \otimes B_{p-i, q-j}^{\prime}
$$

is again a bicomplex. The result follows by iterated application of the Künneth isomorphism. Specifically, we use the induction and have the isomorphism

$$
\begin{aligned}
\left(E^{r} B \otimes E^{r} B^{\prime}\right)_{p, q} & =\bigoplus_{i, j} E_{i, j}^{r}(B) \otimes E_{p-i, q-j}^{r}\left(B^{\prime}\right) \\
& =\bigoplus_{i, j} H\left(E^{r-1}(B), \delta^{r-1}\right)_{i, j} \otimes H\left(E^{r-1}\left(B^{\prime}\right), \delta^{r-1}\right)_{p-i, q-j} \\
& \cong H\left(\left(E^{r-1}(B), \delta^{r-1}\right) \otimes\left(E^{r-1}\left(B^{\prime}\right), \delta^{r-1}\right)\right)_{p, q} \\
& \stackrel{\star}{\cong} H\left(E^{r-1}\left(B \otimes B^{\prime}\right), \delta^{r-1}\right)_{p, q} \\
& =E_{p, q}^{r}\left(B \otimes B^{\prime}\right)
\end{aligned}
$$

where $\star$ is the statement for $r-1$ and the base case is

$$
\begin{aligned}
\left(E^{0} B \otimes E^{0} B^{\prime}\right)_{p, q}= & \bigoplus_{i, j} E_{i, j}^{0}(B) \otimes E_{p-i, q-j}^{0}\left(B^{\prime}\right) \\
& \cong \bigoplus_{i, j} B_{i, j} \otimes B_{p-i, q-j}^{\prime}=\left(B \otimes B^{\prime}\right)_{p, q} \cong E_{p, q}^{0}\left(B \otimes B^{\prime}\right) .
\end{aligned}
$$


Proposition 4.3. Let $X$ and $Y$ be cosimplicial chain complexes. The AlexanderWhitney map

$$
C(X) \otimes C(Y) \rightarrow C(X \otimes Y)
$$

induces an isomorphism

$$
E^{r}(X) \otimes E^{r}(Y) \stackrel{\cong}{\rightrightarrows} E^{r}(X \otimes Y)
$$

for all $r \geq 2$. The inverse is induced from the shuffle map $\nabla$.

Proof. By Lemma 4.2 it is enough to show that the map of bicomplexes $C(X) \otimes$ $C(Y) \rightarrow C(X \otimes Y)$ induces a an isomorphism on page 2 of the associated spectral sequence:

$$
E^{2}(C(X) \otimes C(Y)) \stackrel{\cong}{\rightarrow} E^{2}(C(X \otimes Y)) .
$$

Consider the diagram

$$
\begin{gathered}
C H_{*}(X) \otimes C H_{*}(Y) \stackrel{A W}{\longrightarrow} C\left[H_{*}(X) \otimes H_{*}(Y)\right] \stackrel{\cong}{\|} C H_{*}(X \otimes Y) \\
H_{*} C(X) \otimes H_{*} C(Y) \stackrel{\|}{\longrightarrow} H_{*}[C(X) \otimes C(Y)] \stackrel{H_{*} A W}{\longrightarrow} H_{*} C(X \otimes Y)
\end{gathered}
$$

where the isomorphisms come from the Künneth theorem. It is easy to see that this commutes when we consider $C H_{*}(X)=H_{*} C(X)$ as a subobject of $H_{*}(X)$. The dual Eilenberg-Zilber theorem implies that the top left map $A W$ becomes an isomorphism when we take homology in the horizontal direction. The same thus applies to $H_{*} A W$, implying that the composite

$$
E^{2}(X) \otimes E^{2}(Y) \stackrel{\cong}{\rightarrow} E^{2}(C(X) \otimes C(Y)) \stackrel{E^{2}(A W)}{\longrightarrow} E^{2}(X \otimes Y)
$$

is an isomorphism.

Since $\nabla A W=$ id, the inverse map must be the one induced from $\nabla$.

Remark. One consequence of this proposition is that although

$$
A W: C(Y) \otimes C(Y) \rightarrow C(Y \otimes Y)
$$

is not $\pi$-equivariant, it becomes so on $E^{2}$ (see [6, Theorem 9.3(vii)]). This is because at the level of bicomplexes $\nabla$ is $\pi$-equivariant:

$$
A W \sigma=A W \sigma \mathrm{id}=A W \sigma(\nabla A W)=A W(\nabla \sigma) A W \sim_{2} \text { id } \sigma A W=\sigma A W,
$$

where $\sim_{2}$ indicates that these induce the same map on $E^{2}$.

\section{Isolation of the Rows}

Recall from Figure 7 that the $E^{1}$ page of the spectral sequence for $\mathcal{E}\left(D_{r s t}\right)$ contains three 'horizontal strips' $[-2 s,-s] \times\{2 t\},[-2 s-r,-s-r] \times\{2 t+r-1\}$, and $[-2 s-2 r,-s-r] \times\{2 t+2 r-2\}$ (if $r=\infty$ we only have the first of these) 
which are the only places where $\delta^{1}$ may be nonzero. We introduce a slightly more general class of complexes in this section (basically including the $r=0$ and $r=1$ cases of the middle horizontal strip) to facilitate this computation, and quickly compute the cohomology of the middle horizontal strip. In section 6 we will compute the cohomology of the top and bottom strips.

Fix $s$ and $s^{\prime}$ nonnegative integers and let $\Omega_{s, s^{\prime}}$ be the cochain complex

$$
\Omega_{s, s^{\prime}}=C\left(H_{s}\left(D_{\infty s s}\right) \otimes H_{s^{\prime}}\left(D_{\infty s^{\prime} s^{\prime}}\right)\right) .
$$

When $s=s^{\prime}, \Omega_{s, s}$ has an obvious $\pi$-action and we define

$$
\bar{\Omega}_{s}=\Omega_{s, s} / \pi .
$$

We know from Proposition 2.3 that a basis for $H_{s}\left(D_{\infty s s}\right)$ is given by

$$
\Lambda_{s}^{p}=\{\zeta \mid \zeta:[s] \hookrightarrow[p], \zeta(0)=0\},
$$

and we take $\Lambda_{s}^{p} \times \Lambda_{s^{\prime}}^{p}$ as the preferred basis for $H_{s}\left(D_{\infty s s}^{p}\right) \otimes H_{s^{\prime}}\left(D_{\infty s^{\prime} s^{\prime}}^{p}\right)$. Let

$$
\omega_{s, s^{\prime}}^{p} \subset \Lambda_{s}^{p} \times \Lambda_{s^{\prime}}^{p}
$$

be the set of pairs $\left(\zeta, \zeta^{\prime}\right)$ with $[p]=\operatorname{im} \zeta \cup \operatorname{im} \zeta^{\prime}$.

Lemma 5.1. The set $\omega_{s, s^{\prime}}^{p}$ is a basis for $\Omega_{s, s^{\prime}}^{p}$.

Proof. Recall that if $\zeta \in \Lambda_{s}^{p}, \zeta^{\prime} \in \Lambda_{s^{\prime}}^{p}$, and $k>0$, then $d^{k} \zeta \in \Lambda_{s}^{p+1}$ and $d^{k} \zeta^{\prime} \in \Lambda_{s^{\prime}}^{p+1}$. Thus $d^{k}$ takes basis elements to basis elements for $k>0$. A basis element $\left(\zeta, \zeta^{\prime}\right) \in \Lambda_{s}^{p} \times \Lambda_{s^{\prime}}^{p}$ with $[1, p] \subset \operatorname{im} \zeta \cup \operatorname{im} \zeta^{\prime}$ is not in im $d^{k}$ for any $k>0$, hence is not in $\operatorname{im} d^{1}+\cdots+\operatorname{im} d^{p}$. All other basis elements are in im $d^{k}$ for some $k>0$. Thus the set of elements $\left(\zeta, \zeta^{\prime}\right)$ with $[1, p] \in \operatorname{im} \zeta \cup \operatorname{im} \zeta^{\prime}$ constitutes a basis for a complement of $\operatorname{im} d^{1}+\cdots+\operatorname{im} d^{p}$. The result follows from the definition of conormalization.

Remark. Observe that $\omega_{s, s^{\prime}}^{p}$ is nonempty exactly when $p \in\left[\max \left(s, s^{\prime}\right), s+s^{\prime}\right]$.

Proposition 5.2. Fix $r, s, t$, and consider the spectral sequence for $\mathcal{E}\left(D_{r s t}\right)$. There are maps

$$
\begin{gathered}
\psi_{\text {bot }}: \bar{\Omega}_{s}^{p} \rightarrow E_{-p, 2 t}^{1} \\
\psi_{\text {mid }}: \Omega_{s, s+r}^{p} \rightarrow E_{-p, 2 t+r-1}^{1} \\
\psi_{\text {top }}: \bar{\Omega}_{s+r}^{p} \rightarrow E_{-p, 2 t+2 r-2}^{1}
\end{gathered}
$$

for $p \geq 0$ which are isomorphisms of complexes.

Proof. Assume $t=s$. We have isomorphisms of cosimplicial modules

$$
\begin{aligned}
\left(H_{s}\left(D_{r s s}\right) \otimes H_{s}\left(D_{r s s}\right)\right) / \pi & \rightarrow H_{2 s}\left(\mathcal{E}\left(H_{*}\left(D_{r s s}\right)\right)\right) \\
H_{s}\left(D_{r s s}\right) \otimes H_{s+r-1}\left(D_{r s s}\right) & \rightarrow H_{2 s+r-1}\left(\mathcal{E}\left(H_{*}\left(D_{r s s}\right)\right)\right) \\
\left(H_{s+r-1}\left(D_{r s s}\right) \otimes H_{s+r-1}\left(D_{r s s}\right)\right) / \pi & \rightarrow H_{2 s+2 r-2}\left(\mathcal{E}\left(H_{*}\left(D_{r s s}\right)\right)\right)
\end{aligned}
$$


each given by $\zeta \otimes \zeta^{\prime} \mapsto e_{0} \otimes \zeta \otimes \zeta^{\prime}$. Applying $C$ to the modules on the right gives the nontrivial rows of $E^{1}$.

We now identify the left hand side in the above isomorphisms. The inclusion $D_{r s s} \rightarrow D_{\infty s s}$ induces an isomorphism $H_{s}\left(D_{r s s}\right) \stackrel{\cong}{\rightrightarrows} H_{s}\left(D_{\infty s s}\right)$. Since $H_{s+r} \Delta^{p}=0=H_{s+r-1} \Delta^{p}$ and $\mathrm{sk}_{s-1} \Delta^{p}$ is zero in degree $s+r-1$ and degree $s+r-2$, the long exact sequences associated to the short exact sequences

$$
\begin{gathered}
0 \rightarrow \mathrm{sk}_{s+r-1} \Delta^{p} \rightarrow \Delta^{p} \rightarrow D_{\infty, s+r, s+r}^{p} \rightarrow 0 \\
0 \rightarrow \mathrm{sk}_{s-1} \Delta^{p} \rightarrow \mathrm{sk}_{s+r-1} \Delta^{p} \rightarrow D_{r s s}^{p} \rightarrow 0
\end{gathered}
$$

give isomorphisms

$$
H_{s+r}\left(D_{\infty, s+r, s+r}\right) \stackrel{\cong}{\partial} H_{s+r-1}\left(\operatorname{sk}_{s+r-1} \Delta\right) \stackrel{\cong}{\longrightarrow} H_{s+r-1}\left(D_{r s s}\right) .
$$

Combined, these give isomorphisms

$$
\begin{aligned}
\bar{\Omega}_{s} & \rightarrow C\left(\left(H_{s}\left(D_{r s s}\right) \otimes H_{s}\left(D_{r s s}\right)\right) / \pi\right) \\
\Omega_{s, s+r} & \rightarrow C\left(H_{s}\left(D_{r s s}\right) \otimes H_{s+r-1}\left(D_{r s s}\right)\right) \\
\bar{\Omega}_{s+r, s+r} & \rightarrow C\left(\left(H_{s+r-1}\left(D_{r s s}\right) \otimes H_{s+r-1}\left(D_{r s s}\right)\right) / \pi\right) .
\end{aligned}
$$

Theorem 5.3. The cohomology of $\Omega_{s, s^{\prime}}$ is

$$
H^{n} \Omega_{s, s^{\prime}}= \begin{cases}\mathbb{k} & \text { if } n=s+s^{\prime} \\ 0 & \text { otherwise. }\end{cases}
$$

Proof. Notice that $H_{*} D_{\infty s s}$ is concentrated in degree $s$ by Proposition 2.3 So

$$
\begin{aligned}
H^{*} \Omega_{s, s^{\prime}} & =H^{*} C\left(H_{*} D_{\infty s s} \otimes H_{*} D_{\infty s^{\prime} s^{\prime}}\right) \\
& =E^{2}\left(H_{*} D_{\infty s s} \otimes H_{*} D_{\infty s^{\prime} s^{\prime}}\right) \\
& \cong E^{2}\left(H_{*} D_{\infty s s}\right) \otimes E^{2}\left(H_{*} D_{\infty s^{\prime} s^{\prime}}\right)
\end{aligned}
$$

where the last isomorphism is by Proposition 4.3. The result follows from the computation of $E^{1}\left(D_{\infty s s}\right)$ in Proposition 2.4.

\section{Cohomology of $\bar{\Omega}$}

Fix $s \geq 0$. In this section we employ a short exact sequence in order to compute the cohomology of $\bar{\Omega}_{s}$. Let $A=\operatorname{ker}\left(\Omega_{s, s} \rightarrow \bar{\Omega}_{s}\right)$ and consider the exact sequence

$$
0 \rightarrow A \rightarrow \Omega_{s, s} \rightarrow \bar{\Omega}_{s} \rightarrow 0 .
$$

We begin by identifying the complex $A$ and studying its cohomology. 
Observe that for $p>s$, if $\left(\zeta, \zeta^{\prime}\right) \in \omega_{s, s}^{p}$ then $\sigma\left(\zeta, \zeta^{\prime}\right) \neq\left(\zeta, \zeta^{\prime}\right)$. Thus $\Omega_{s, s}^{p}$ is a free $\mathbb{k} \pi$-module for $p>s$, so

$$
\operatorname{ker}\left(\Omega_{s, s}^{p} \rightarrow \bar{\Omega}_{s}^{p}\right)=A^{p}=(1+\sigma) \Omega_{s, s}^{p} .
$$

Furthermore, $\omega_{s, s}^{s}=\left\{\left(\operatorname{id}_{[s]}, \operatorname{id}_{[s]}\right)\right\}$, so $A^{s}=0$.

We have now identified $A$ as the image of the map

$$
\Omega_{s, s} \stackrel{1+\sigma}{\longrightarrow} \Omega_{s, s}
$$

The kernel of this map,

$$
\Upsilon=\operatorname{ker}\left(1+\sigma: \Omega_{s, s} \rightarrow A\right)
$$

will be of independent interest (see [9, Proposition 2.2]). For now, notice that $\Upsilon^{p}=(1+\sigma) \Omega_{s, s}^{p}=A^{p}$ for $p>s$ since $\Omega_{s, s}^{p}$ is $\mathbb{k} \pi$-free. This implies that

$$
H^{p} \Upsilon=H^{p} A \quad p \geq s+2
$$

Using this, we have the following lemma.

Lemma 6.1. Consider the inclusion $\Upsilon \rightarrow \Omega_{s, s}$. If $s \geq 2$, then the induced map

$$
H^{2 s}(\Upsilon) \rightarrow H^{2 s}\left(\Omega_{s, s}\right)
$$

is zero. As a consequence, the inclusion $A \rightarrow \Omega_{s, s}$ induces the zero map $H^{2 s}(A) \rightarrow H^{2 s}\left(\Omega_{s, s}\right)$.

Proof. By assumption, $s \geq 2$, so $H^{2 s} \Upsilon=H^{2 s} A$. The short exact sequence

$$
0 \rightarrow \Upsilon \rightarrow \Omega_{s, s} \rightarrow A \rightarrow 0
$$

induces the exact sequence

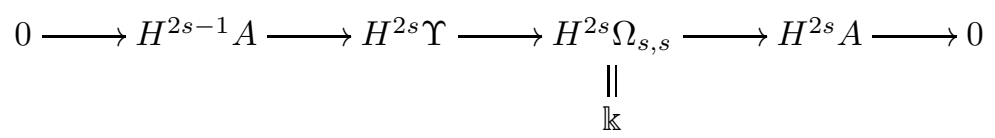

since $H^{2 s} \Omega_{s, s}=\mathbb{k}$ by Theorem 5.3 and $\Upsilon^{2 s+1}=0$. Seeking a contradiction, suppose that $H^{2 s} \Upsilon \rightarrow H^{2 s} \Omega_{s, s}$ is nonzero. Then $0=H^{2 s} A$, but since $s \geq 2$ we have $H^{2 s} A=H^{2 s} \Upsilon$. This contradicts our assumption.

Proposition 6.2. Fix $s \geq 0$. We have

$H^{p}(A)=\left\{\begin{array}{ll}\mathbb{k} & \text { for } p \in[s+1,2 s], s>0, \\ 0 & \text { else }\end{array} H^{p}(\Upsilon)= \begin{cases}\mathbb{k} & \text { for } p \in[s+2,2 s], s>1, \\ \mathbb{k} \quad \text { for } p=0=s, \\ 0 \quad \text { else. }\end{cases}\right.$ 
Proof. We use the long exact cohomology sequence associated to the short exact sequence

$$
0 \rightarrow \Upsilon \rightarrow \Omega_{s, s} \rightarrow A \rightarrow 0
$$

as well as Theorem 5.3. which says that $H^{2 s} \Omega_{s, s}=\mathbb{k}$ and $H^{p} \Omega_{s, s}=0$ for $p \neq 2 s$.

If $s=0$ then $A=0$, so $\Upsilon=\Omega_{0,0}$ and the result is obvious.

If $s=1$, then $A^{p}=0$ for $p \neq 2$ and $A^{2} \neq 0$, so exactness of

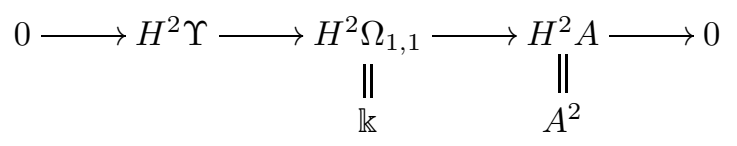

implies $H^{2} \Upsilon=0$ and $H^{2} A=\mathbb{k}$.

Suppose that $s \geq 2$. Examine the exact sequence

$$
0 \rightarrow H^{2 s-1} A \rightarrow H^{2 s} \Upsilon \rightarrow H^{2 s} \Omega_{s, s} \rightarrow H^{2 s} A \rightarrow 0 .
$$

By Lemma 6.1, $H^{2 s} \Upsilon \rightarrow H^{2 s} \Omega_{s, s}$ is zero. Thus we have $\mathbb{k}=H^{2 s} \Omega_{s, s}=H^{2 s} A$, and $H^{2 s-1} A \rightarrow H^{2 s} \Upsilon=H^{2 s} A=\mathbb{k}$ is an isomorphism. For $p<2 s$, we have that

$$
0 \rightarrow H^{p-1} A \rightarrow H^{p} \Upsilon \rightarrow 0
$$

is exact, so for $s+2 \leq p<2 s$ we have

$$
H^{p-1} A=H^{p} \Upsilon=H^{p} A=\mathbb{k} .
$$

To finish this case, notice that $A^{p-1}=0$ for $p-1 \leq s$, so $0=H^{p-1} A=H^{p} \Upsilon$ for $p \leq s+1$.

Theorem 6.3. The cohomology of $\bar{\Omega}_{s}$ is

$$
H^{n} \bar{\Omega}_{s}= \begin{cases}\mathbb{k} & n \in[s, 2 s] \\ 0 & \text { otherwise }\end{cases}
$$

Proof. We use the exact sequence

$$
0 \rightarrow A \rightarrow \Omega_{s, s} \rightarrow \bar{\Omega}_{s} \rightarrow 0,
$$

Theorem 5.3. and Proposition 6.2. Notice immediately that $H^{i-1} \bar{\Omega}_{s} \cong H^{i} A$ for $i<2 s$, so we are reduced to analyzing the exact sequence

$$
0 \rightarrow H^{2 s-1} \bar{\Omega}_{s} \rightarrow H^{2 s} A \rightarrow H^{2 s} \Omega_{s, s} \rightarrow H^{2 s} \bar{\Omega}_{s} \rightarrow 0 .
$$

We merely need to show that $H^{2 s} A \rightarrow H^{2 s} \Omega_{s, s}$ is always zero. When $s \geq 2$, this is just Lemma 6.1. If $s=1$, and $\alpha \in A^{2}=\Upsilon^{2}$ is a cycle, then $\alpha$ is a boundary in $\Upsilon$ since $H^{2} \Upsilon=0$, hence $\alpha$ is a boundary in $H^{2}\left(\Omega_{1,1}\right)$. Finally, $H^{0} A \rightarrow H^{0} \Omega_{0,0}$ is trivially zero since $A=0$ when $s=0$. 


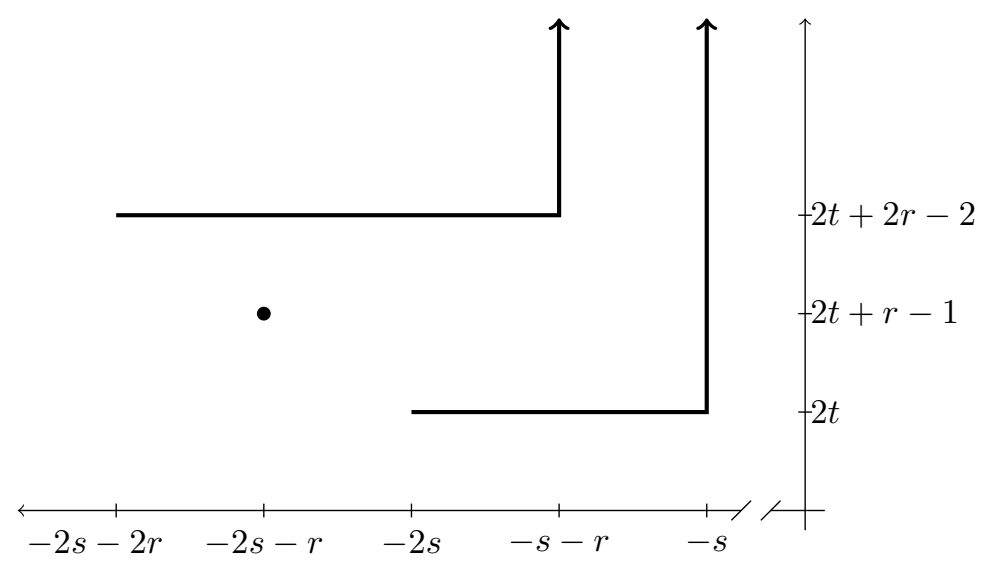

Figure 8: $E^{2}\left(\mathcal{E}\left(D_{r s t}\right)\right)$

\section{The $E^{2}$ page}

We now record the $E^{2}$ page of the spectral sequence. See Figure 8

Theorem 7.1. Let $2 \leq r \leq \infty$. Each bidegree in the $E^{2}$ page of the spectral sequence for $\mathcal{E}\left(D_{\text {rst }}\right)$ is either 0 or $\mathbb{k}$. The nontrivial terms are in

$$
\begin{gathered}
\{-s\} \times[2 t, \infty) \\
{[-2 s,-s-1] \times\{2 t\}}
\end{gathered}
$$

and, if $r<\infty$, in

$$
\begin{gathered}
\{-s-r\} \times[2 t+2 r-2, \infty) \\
\{-2 s-r\} \times\{2 t+r-1\} \\
{[-2 s-2 r,-s-r-1] \times\{2 t+2 r-2\} .}
\end{gathered}
$$

Proof. Theorem 3.3 gives the $E^{1}$ page. The structure of that page gives the ranges $\{-s\} \times(2 t, \infty)$ and $\{-s-r\} \times(2 t+2 r-2, \infty)$. Theorems 5.3 and 6.3 combine with Proposition 5.2 to give the rest.

Notice that when $r=\infty$, the structure of the $E^{2}$ page implies that all further differentials are zero, so

$$
E_{-p, q}^{\infty}= \begin{cases}\mathbb{k} & \text { if } q=2 t \text { and }-p \in[-2 s,-s], \\ \mathbb{k} & \text { if } p=s \text { and } q \geq 2 t \\ 0 & \text { else. }\end{cases}
$$

We will compute the differentials for finite $r$ in the next two sections. 


\section{8. $E^{\infty}=0$ when $r<\infty$}

Let $D=D_{r s s}$ for $r<\infty$. The goal of this section is contained in its title: we wish to show that $E^{\infty}(\mathcal{E}(D))=0$.

Lemma 8.1. If $r$ is finite, then the bicomplex $C(D \otimes D)$ is finite.

Proof. The vector space $C(D \otimes D)_{m}^{p}$ has a basis consisting of elements $\varepsilon \otimes \varepsilon^{\prime}$ where $\varepsilon:[q] \hookrightarrow[p], \varepsilon^{\prime}:\left[q^{\prime}\right] \hookrightarrow[p], q+q^{\prime}=m$, and $[1, p] \subset \operatorname{im} \varepsilon \cup \operatorname{im} \varepsilon^{\prime}$. Furthermore, since we are working in $D_{r s s}$ we require that $q, q^{\prime} \in[s, s+r-1]$. Thus we see that $C(D \otimes D)_{m}^{p}$ is zero unless $m \in[2 s, 2(s+r-1)]$ and $p \in$ $[s, 2(s+r)]$, so $C(D \otimes D)$ is bounded. Furthermore, each $C(D \otimes D)_{m}^{p}$ is finite.

Proposition 8.2. For $r$ finite we have $H \operatorname{Tot} C(D \otimes D)=0$.

Proof. Lemma 8.1 implies convergence, so we have

$$
H \operatorname{Tot} C(D \otimes D) \cong \operatorname{Tot} E^{\infty}(D \otimes D) \cong \operatorname{Tot}\left[E^{\infty}(D) \otimes E^{\infty}(D)\right]
$$

by Proposition 4.3. We saw in section 2.2 that $E^{\infty}\left(D_{r s s}\right)=0$ for $r<\infty$.

We will need similar algebraic convergence results for $\mathcal{E}\left(D_{r s t}\right)$.

Theorem 8.3. Let $2 \leq r \leq \infty$. Then

$$
H_{*} \operatorname{Tot} C\left(\mathcal{E}\left(D_{r s t}\right)\right) \cong \operatorname{Tot} E^{\infty}\left(\mathcal{E}\left(D_{r s t}\right)\right) .
$$

Proof. Write $B=C\left(\mathcal{E}\left(D_{r s t}\right)\right)$. Note that $\operatorname{Tot} B$ is the completion (as a filtered complex) of the sum totalization $\left(\operatorname{Tot}^{\oplus} B\right)_{m}=\oplus_{p+q=m} B_{p, q}$. Reindexing so that we have a fourth quadrant, cohomological bicomplex with vertical differential $d_{v}$ and horizontal differential $d_{h}$, we have conditional convergence of our spectral sequence by [3, Theorem 10.1].

Since each group $E_{-p, q}^{1}$ is a finite $\mathbb{k}$-module, only finitely many differentials $\delta^{j}: E_{-p, q}^{j} \rightarrow E_{-p-j, q+j-1}^{j}$ may be nonzero. Thus we have strong convergence of our spectral sequence by [3, Theorem 7.1] and the Remark immediately following it.

The following proposition works over any ground ring and, in particular, gives

$$
C\left(W \otimes_{\pi}(D \otimes D)\right) \cong W^{v} \otimes_{\pi} C(D \otimes D),
$$

where $W^{v}$ is the bicomplex concentrated on the $y$-axis with $W_{0, *}^{v}=W_{*}$.

Proposition 8.4. Let $X_{*}$ be a chain complex and let $X_{*}^{v}$ be the bicomplex which has $X$ as its zeroth column. If $Y_{*}^{\bullet}$ is a cosimplicial chain complex then

$$
C\left(X_{*} \otimes Y_{*}^{\bullet}\right) \cong X_{*}^{v} \otimes C\left(Y_{*}^{\bullet}\right) .
$$


Finiteness of $C(D \otimes D)$ allows us to conclude that

$$
\operatorname{Tot} C(\mathcal{E}(D))=\operatorname{Tot} C\left(W \otimes_{\pi}(D \otimes D)\right) \cong W \otimes_{\pi} \operatorname{Tot} C(D \otimes D) .
$$

Furthermore, the functor $W \otimes_{\pi}-$ preserves quasi-isomorphism.

Proposition 8.5. Suppose that $L \rightarrow L^{\prime}$ is a map of nonnegatively-graded $\mathbb{k} \pi$ complexes which induces an isomorphism in homology. Then

$$
H\left(W \otimes_{\pi} L\right) \rightarrow H\left(W \otimes_{\pi} L^{\prime}\right)
$$

is an isomorphism as well.

Proof. The Künneth spectral sequence (see [17, Theorem 2.20])

$$
E_{2}^{p, q}=\bigoplus_{s+t=q} \operatorname{Tor}_{\mathbb{k} \pi}^{p}\left(H^{s}(W), H^{t}(L)\right) \Rightarrow H\left(W \otimes_{\pi} L\right)
$$

is a first-quadrant spectral sequence, so it converges. The map $L \rightarrow L^{\prime}$ induces an isomorphism on $E_{2}$.

Proposition 8.6. For $r$ finite we have $\operatorname{Tot} E^{\infty}\left(\mathcal{E}\left(D_{r s t}\right)\right)=H \operatorname{Tot} C\left(\mathcal{E}\left(D_{r s t}\right)\right)=$ 0 .

Proof. We already saw that $\operatorname{Tot} C\left(\mathcal{E}\left(D_{\text {rst }}\right)\right) \cong W \otimes_{\pi} \operatorname{Tot} C\left(D_{\text {rst }} \otimes D_{\text {rst }}\right)$, so we have $H \operatorname{Tot} C\left(\mathcal{E}\left(D_{r s t}\right)\right)=0$ by Propositions 8.2 and 8.5 . The spectral sequence converges by Theorem 8.3 .

\section{All Other Differentials Are Automatic}

A spectral sequence with $E^{2}$ page of the form of Theorem 7.1 with $E^{\infty}=0$ can only have one pattern of differential, which we give a rough picture of in Figure 9. We need only consider differentials $\delta^{j}: E_{p, q}^{j} \rightarrow E_{p-j, q+j-1}^{j}$ for $j \geq 2$.

Proposition 9.1. The following differentials in the spectral sequence associated to $\mathcal{E}\left(D_{\text {rst }}\right)$ are nontrivial:

$$
\begin{array}{rlrl}
\delta^{r}: E_{-2 s-r, 2 t+r-1}^{r} & \rightarrow E_{-2 s-2 r, 2 t+2 r-2}^{r} & & \\
\delta^{2 r-1}: E_{p, 2 t}^{2 r-1} & \rightarrow E_{p-2 r+1,2 t+2 r-2}^{2 r-2} & & p \in[-2 s,-s-1] \\
\delta^{2 r-1-b}: E_{-s, 2 t+b}^{2 r-1-b} & \rightarrow E_{b+1-2 r-s, 2 t+2 r-2}^{2 r-1-b} & b & b[0, r-2] \\
\delta^{r}: E_{-s, 2 t+b}^{r} & \rightarrow E_{-s-r, 2 t+b+r-1}^{r} & b & b[r-1, \infty)
\end{array}
$$

Proof. First we look at the 'top row' $[-2 s-2 r,-s-r] \times\{2 t+2 r-2\}$. All differentials $\delta^{j}$ out of $E_{p, q}^{j}$ for $(p, q) \in[-2 s-2 r,-s-r] \times\{2 t+2 r-2\}$ must 
be zero. We list all possibilities for differentials mapping to this row which have the potential to be nontrivial:

$$
\begin{aligned}
\delta^{2 r-1} & : E_{p, 2 t}^{2 r-1} \rightarrow E_{p-2 r+1,2 t+2 r-2}^{2 r-1} \\
\delta^{r} & : E_{-2 s-r, 2 t+r-1}^{r} \rightarrow E_{-2 s-2 r, 2 t+2 r-2}^{r} \\
\delta^{j} & : E_{-s, 2 t+2 r-j-1}^{j} \rightarrow E_{-s-j, 2 t+2 r-2}^{j}
\end{aligned}
$$

where

$$
\begin{aligned}
& p \in[-2 s,-s] \leadsto p-2 r+1 \in[-2 s-2 r+1,-2 r-s+1] \\
& j \in[r, 2 r-2] \leadsto-s-j \in[2-s-2 r,-s-r] .
\end{aligned}
$$

But we have that

$[-2 s-2 r,-s-r]=[-2 s-2 r+1,-2 r-s+1] \sqcup\{-2 s-2 r\} \sqcup[2-2 r-s,-s-r]$,

so each map listed above must have rank 1.

This leaves us only with the leftmost column

$$
\{-s-r\} \times[2 t+2 r-1, \infty)
$$

and part of the rightmost column $\{-s\} \times[2 t+r, \infty)$ still unaccounted for. Then it is obvious that there is only one possibility:

$$
\delta^{r}: E_{-s, q}^{r} \rightarrow E_{-s-r, q+r-1}^{r} \quad q \in[2 t+r, \infty) .
$$

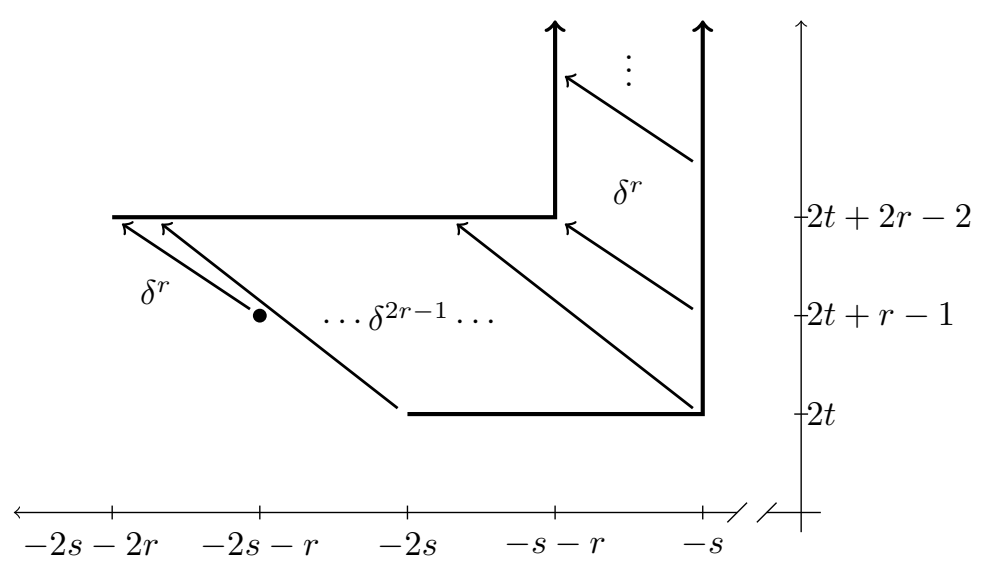

Figure 9: Differentials in the spectral sequence associated to $\mathcal{E}\left(D_{\text {rst }}\right)$ 
Corollary 9.2. Let $E_{*, *}^{*}$ be the spectral sequence associated with $\mathcal{E}\left(D_{r s t}\right)$. We record when various bidegrees become zero; they each contain a copy of $\mathbb{k}$ on the previous page. First for the lower right portion

$$
\begin{aligned}
E_{p, 2 t}^{2 r} & =0 & & p \in[-2 s,-s] \\
E_{-s, v}^{2 t+2 r-v} & =0 & & v \in[2 t+1,2 t+r-1] \\
E_{-s, q}^{r+1} & =0 & & q \in[2 t+r, \infty)
\end{aligned}
$$

then for the upper left portion

$$
\begin{aligned}
E_{-2 s-2 r, 2 t+2 r-2}^{r+1}=0 & & \\
E_{p, 2 t+2 r-2}^{2 r}=0 & & p \in[-2 s-2 r+1,-2 r-s+1] \\
E_{p, 2 t+2 r-2}^{-s-p+1}=0 & & p \in[-2 r-s+2,-s-r] \\
E_{-s-r, q}^{r+1}=0 & & q \in[2 t+2 r-1, \infty)
\end{aligned}
$$

and finally

$$
E_{-2 s-r, 2 t+r-1}^{r+1}=0
$$

Remark. In the spectral sequence associated to $\mathcal{E}\left(D_{r s t}\right)$ we have $E^{2 r}=0$.

\section{Products and Operations on Cycles}

This section is a bit of a warm-up for what will come. The first goal is to define the (external) product in the spectral sequence of a cosimplicial chain complex $Y$ and show that it is commutative. We define external operations for $r$-cycles and show that the bottom operation agrees with the external square.

In general, if $Y$ is a cosimplicial chain complex equipped with a multiplication $Y \otimes Y \rightarrow Y$, then there is a product $E^{r}(Y) \otimes E^{r}(Y) \rightarrow E^{r}(Y)$ which is a derivation for $\delta^{r}$, coming from

$$
C(Y) \otimes C(Y) \stackrel{A W}{\rightarrow} C(Y \otimes Y) \rightarrow C(Y)
$$

where $A W$ is the Alexander-Whitney map from (11). In our setting, we start with a cosimplicial map

$$
\theta: \mathcal{E}(Y) \rightarrow Y
$$

and obtain a product by precomposition with the composite

$$
\kappa: Y \otimes Y=\mathbb{k} \otimes Y \otimes Y \stackrel{1 \mapsto e_{0}}{\longrightarrow} W \otimes Y \otimes Y \rightarrow W \otimes_{\pi}(Y \otimes Y)=\mathcal{E}(Y) .
$$

The following proposition shows that products $E^{r}(Y) \otimes E^{r}(Y) \rightarrow E^{r}(Y)$ obtained from

$$
Y \otimes Y \stackrel{\kappa}{\rightarrow} \mathcal{E}(Y) \stackrel{\theta}{\rightarrow} Y
$$

are commutative for $r \geq 2$. 
Proposition 10.1. Let $Y$ be a cosimplicial chain complex and $r \geq 2$. The external product

$$
\mu_{r}: E^{r}(Y) \otimes E^{r}(Y) \stackrel{A W}{\rightarrow} E^{r}(Y \otimes Y) \rightarrow E^{r}(\mathcal{E}(Y))
$$

is commutative.

Proof. Since $A W$ becomes $\pi$-equivariant starting at $E^{2}$ (see the remark on page 20), we can reduce the problem to showing that the following holds on $E^{2}$ :

$$
\mu_{2} \sigma=\kappa A W \sigma=\kappa \sigma A W \stackrel{?}{=} \kappa A W=\mu_{2} .
$$

The equality $\mu_{r} \sigma=\mu_{r}$ then follows for all $r \geq 2$.

Thus we merely need to show that $\kappa \sigma=\kappa$ on $E^{2}$. This is actually true on $E^{1}$. Consider $v \in Z_{-s}^{1}(Y \otimes Y) \subset \operatorname{Tot} C(Y \otimes Y)$, then we have the formula

$$
\partial\left(e_{1} \otimes v\right)=(1+\sigma) e_{0} \otimes v+e_{1} \otimes \partial v
$$

in $\operatorname{Tot}\left[W^{v} \otimes_{\pi} C(Y \otimes Y)\right]=\operatorname{Tot} C(\mathcal{E}(Y))$. Notice that

$$
\partial\left(e_{1} \otimes v\right) \in \partial F^{-s}=\partial Z_{-s+1-1}^{0} \subset B_{-s}^{1}
$$

and

$$
e_{1} \otimes \partial v \in F^{-s-1}=Z_{-s-1}^{0} \subset B_{-s}^{1} .
$$

Furthermore,

$$
(1+\sigma) e_{0} \otimes v=(\kappa+\kappa \sigma) v
$$

so $\kappa=\kappa \sigma$ on $E^{1}$.

We now define external operations on $r$-cycles using the universal property of $D_{\text {rst }}$. The idea is that the lower 'I' in the spectral sequence for $\mathcal{E}\left(D_{\text {rst }}\right)$ should map to the external operations. We saw in $\$$ that the $2^{\text {nd }}$ page is the same as the $r^{\text {th }}$ page in this spectral sequence.

Theorem 7.1 says that $E_{p, q}^{2}=E_{p, q}^{r}$ is either $\mathbb{k}$ or 0 ; in the former case we write

$$
0 \neq u_{p, q} \in E_{p, q}^{2}=E_{p, q}^{r}=\mathbb{k}
$$

for the generator.

Definition. We define functions

$$
\tilde{Q}^{m}: Z^{r}(Y) \rightarrow E^{r}(\mathcal{E}(Y))
$$

as follows. For $y \in Z_{-s, t}^{r}(Y)$, we let

$$
\begin{aligned}
& \tilde{Q}_{v}^{m}(y)=E^{r}\left(\mathcal{E}\left(\Theta_{y}\right)\right)\left(u_{-s, m+t}\right) \quad m \geq t \\
& \tilde{Q}_{h}^{m}(y)=E^{r}\left(\mathcal{E}\left(\Theta_{y}\right)\right)\left(u_{m-s-t, 2 t}\right) \quad m \in[t-s, t]
\end{aligned}
$$

which are all classes of $E^{r}(\mathcal{E}(Y))$. Here $\Theta_{y}$ is the map from Proposition 2.5 and $u_{p, q}$ is the universal class from (14). 
The idea is that the lower ' $\mathrm{I}$ ' in the spectral sequence for $\mathcal{E}\left(D_{r s t}\right)$ should map to the external operations of $y \in Z_{-s, t}^{r}$.

Remark. Recall that on $E^{r}, \Theta_{y}$ only depends on the class of $y$ in $E^{r}$, rather than on $y$ itself. The situation is much more subtle for $\mathcal{E}\left(\Theta_{y}\right)$, and, at $E^{r}$, this map does depend on the specific choice of $r$-cycle.

Notice that an $r$-cycle is, in particular, an $(r-1)$-cycle. Let us now compare the answers we get by considering an $r$-cycle in these two ways.

Proposition 10.2. Let $r>2$, and suppose $y \in Z_{-s, t}^{r}(Y)$. Write $y_{r}$ for $y$ considered as an element of $Z^{r}$ and $y_{r-1} \in Z^{r-1}$ for $y$ considered as an $r-1$ cycle. Then

$$
\tilde{Q}^{m}\left(y_{r}\right)=\left[\tilde{Q}^{m}\left(y_{r-1}\right)\right]_{r} .
$$

Before beginning the proof, notice that we can easily compare the two constructions because

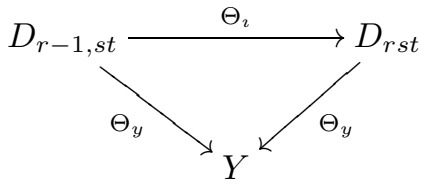

commutes.

We will need the following lemma. It says that if we consider the inclusion $\Theta_{\imath}: D_{r s t} \rightarrow D_{\infty s t}$, where $\imath$ is the class defined in (8), then $E^{2}\left(\mathcal{E}\left(\Theta_{\imath}\right)\right)$ is an injection when restricted to the bottom ' $\mathrm{I}$ '.

Lemma 10.3. Consider the inclusion $\Theta_{\imath}: D_{r s t} \rightarrow D_{\infty s t}$. The map $E^{2}\left(\mathcal{E}\left(\Theta_{\imath}\right)\right)$ is an isomorphism in the bidegrees $[-2 s,-s] \times\{2 t\}$ and $\{-s\} \times[2 t, \infty)$.

Proof. On $E^{1}$, the map $\mathcal{E}\left(\Theta_{\imath}\right)$ is an isomorphism in this range.

Proof of Proposition 10.2. A special case of diagram (16) is when $Y=D_{\infty s t}$ and $y=\imath$. Combined with Lemma 10.3, this tells us that in the spectral sequence, $\mathcal{E}\left(D_{r-1, s t}\right) \rightarrow \mathcal{E}\left(D_{r s t}\right)$ takes the lower 'I' to the lower 'I'. Furthermore, the following commutes,

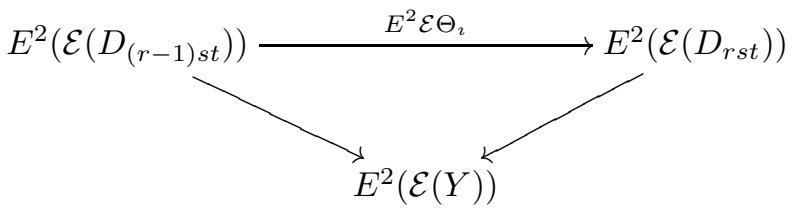

which implies that the representatives on the second page of $\tilde{Q}^{m}\left(y_{r}\right)$ and $\tilde{Q}^{m}\left(y_{r-1}\right)$ are the same. The result follows. 


\subsection{Bottom Operation is the Square}

We now show that the bottom operation coincides with the squaring operation. In particular, since the external product is commutative, this shows that the bottom operation is additive.

Lemma 10.4. Let $r \geq 2$. In the case of the universal example $D_{r s t}$,

$$
\mu_{r}(\imath \otimes \imath) \neq 0
$$

where

$$
\mu_{r}: E^{r}\left(D_{r s t}\right) \otimes E^{r}\left(D_{r s t}\right) \rightarrow E^{r}\left(\mathcal{E}\left(D_{r s t}\right)\right)
$$

is the external multiplication.

Proof. First notice that we can factor the external multiplication as

$$
E^{j}\left(D_{r s t}\right) \otimes E^{j}\left(D_{r s t}\right) \stackrel{\cong}{\rightrightarrows} E^{j}\left(D_{r s t} \otimes D_{r s t}\right) \rightarrow E^{j}\left(\mathcal{E}\left(D_{r s t}\right)\right),
$$

with the first arrow an isomorphism when $j \geq 2$ by Lemma 4.2. We will prove that

$$
E^{2}\left(D_{r s t} \otimes D_{r s t}\right) \rightarrow E^{2}\left(\mathcal{E}\left(D_{r s t}\right)\right)
$$

is nontrivial; the result then follows by examining the spectral sequence for $\mathcal{E}\left(D_{r s t}\right)$ since nothing can hit the element in bidegree $(-2 s, 2 t)$.

The vertical maps in the following commutative diagram are nontrivial, where $D_{r s t} \rightarrow D_{\infty s t}$ is the inclusion.

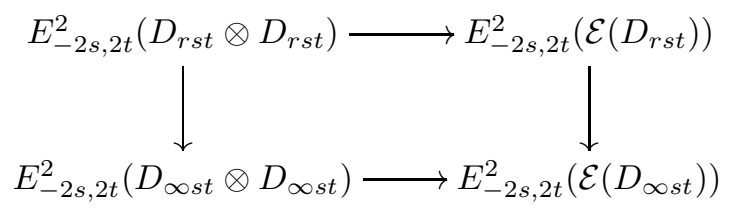

We must see that the bottom map is nontrivial. To do so, note that the diagram

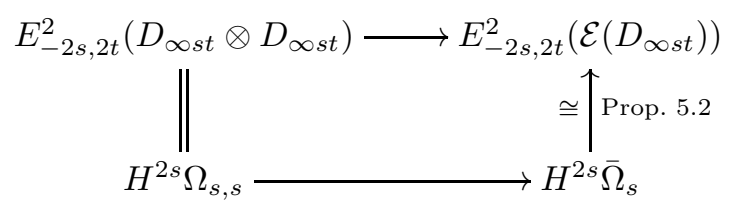

also commutes (where $\Omega_{s, s}$ and $\bar{\Omega}_{s}$ are defined in (12, 13)), and the first map in the exact sequence

$$
H^{2 s} A \rightarrow H^{2 s} \Omega_{s, s} \rightarrow H^{2 s} \bar{\Omega}_{s} \rightarrow 0
$$

is zero by Lemma 6.1, so $H^{2 s} \Omega_{s, s} \rightarrow H^{2 s} \bar{\Omega}_{s}$ is an isomorphism.

Proposition 10.5. Let $y \in Z_{-s, t}^{r}(Y)$, where $r \geq 2$. Then

$$
\mu_{r}([y],[y])=\tilde{Q}^{t-s}(y)
$$


Proof. Let $\Theta_{y}: D_{r s t} \rightarrow Y$ be the representing map from Proposition 2.5. Then

$$
\tilde{Q}^{t-s}(y)=E^{r}\left(\mathcal{E}\left(\Theta_{y}\right)\right)\left(u_{-2 s, 2 t}\right)=E^{r}\left(\mathcal{E}\left(\Theta_{y}\right)\right)\left(\mu_{r}(\imath \otimes \imath)\right)
$$

by the preceding lemma, where $u_{p, q}$ is the universal class from (14). Since $\mu_{r}$ : $E^{r}(-) \otimes E^{r}(-) \Rightarrow E^{r}(\mathcal{E}(-))$ is a natural transformation, we have a commutative diagram

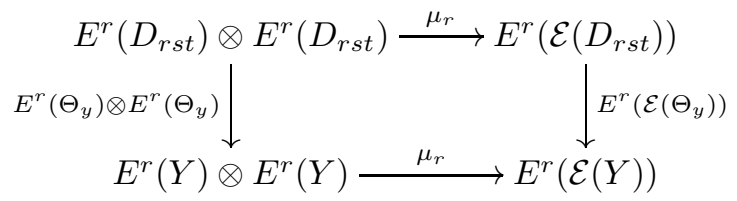

so

$$
\tilde{Q}^{t-s}(y)=\mu_{r}\left(E^{r}\left(\Theta_{y}\right) \otimes E^{r}\left(\Theta_{y}\right)(\imath \otimes \imath)\right)=\mu_{r}([y],[y]) .
$$

There are two consequences to this proposition. The first is that

$$
\tilde{Q}^{t-s}: Z_{-s, t}^{r}(Y) \rightarrow E^{r}(\mathcal{E}(Y))
$$

is additive. This follows from commutativity of $\mu_{r}$. Second, $\tilde{Q}^{t-s}$ induces a homomorphism

$$
Q^{t-s}: E_{-s, t}^{r}(Y) \rightarrow E^{r}(\mathcal{E}(Y))
$$

since $\mu_{r}$ only depends on the $E^{r}$-class of a given $r$-cycle.

\section{Additivity and Sums of Bousfield-Kan Examples}

The goal of this section is to prove the following proposition for $m>t-s$.

Proposition 11.1 (Additivity). Let $r \geq 2$. The functions

$$
\begin{array}{lll}
\tilde{Q}_{v}^{m}: Z_{-s, t}^{r}(Y) \rightarrow E_{-s, m+t}^{r}(\mathcal{E}(Y)) & & m \geq t \\
\tilde{Q}_{h}^{m}: Z_{-s, t}^{r}(Y) \rightarrow E_{m-s-t, 2 t}^{r}(\mathcal{E}(Y)) & & m \in[t-s, t]
\end{array}
$$

are homomorphisms.

Let $x, y \in Z_{-s, t}^{r}(Y)$. The following diagram commutes:

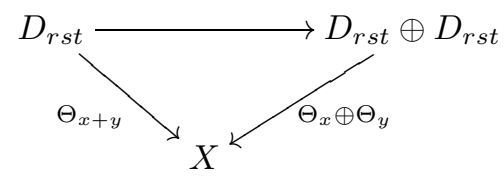

where the top map is the diagonal. This suggests that analyzing the spectral sequence for $\mathcal{E}\left(D_{r s t} \oplus D_{r s t}\right)$ may be helpful in understanding additivity. We will need greater generality later, so we now investigate the spectral sequence associated to $\mathcal{E}\left(D_{r s t} \oplus D_{r^{\prime} s^{\prime} t^{\prime}}\right)$. 
Lemma 11.2. Let $X$ and $Y$ be cosimplicial chain complexes. Then

$$
\mathcal{E}(X \oplus Y) \cong \mathcal{E}(X) \oplus \mathcal{E}(Y) \oplus(W \otimes X \otimes Y)
$$

via

$$
e_{n} \otimes(x+y) \otimes\left(x^{\prime}+y^{\prime}\right) \mapsto \begin{gathered}
e_{n} \otimes x \otimes x^{\prime}+e_{n} \otimes y \otimes y^{\prime} \\
+e_{n} \otimes x \otimes y^{\prime}+\sigma e_{n} \otimes x^{\prime} \otimes y
\end{gathered}
$$

and the obvious inclusions of the first two summands along with the inclusion

$$
\begin{aligned}
W \otimes X \otimes Y & \rightarrow W \otimes_{\pi}((X \oplus Y) \otimes(X \oplus Y)) \\
e_{n} \otimes x \otimes y & \mapsto e_{n} \otimes x \otimes y \\
\sigma e_{n} \otimes x \otimes y & \mapsto \sigma e_{n} \otimes x \otimes y=e_{n} \otimes y \otimes x .
\end{aligned}
$$

Proof. We may work with a fixed cosimplicial degree, so we prove the corresponding statement for chain complexes. If $A$ and $B$ are chain complexes, then

$$
((A \oplus B) \otimes(A \oplus B))=(A \otimes A) \oplus(B \otimes B) \oplus(A \otimes B \oplus B \otimes A)
$$

as $\mathbb{k} \pi$-modules. Since $A \otimes B \oplus B \otimes A$ is a free $\pi$-module, we see

$$
\begin{aligned}
W \otimes_{\pi}((A \oplus B) \otimes & (A \oplus B)) \\
& =\left(W \otimes_{\pi}(A \otimes A)\right) \oplus\left(W \otimes_{\pi}(B \otimes B)\right) \oplus(W \otimes A \otimes B) .
\end{aligned}
$$

As mentioned above, we now consider the special case

$$
\mathcal{E}\left(D_{r s t} \oplus D_{r^{\prime} s^{\prime} t^{\prime}}\right) \cong \mathcal{E}\left(D_{r s t}\right) \oplus \mathcal{E}\left(D_{r^{\prime} s^{\prime} t^{\prime}}\right) \oplus\left(W \otimes D_{r s t} \otimes D_{r^{\prime} s^{\prime} t^{\prime}}\right) .
$$

Conormalization is an additive functor, as is the functor which takes a bicomplex to its associated spectral sequence, so we see that we need only compute the spectral sequence for

$$
W \otimes D_{r s t} \otimes D_{r^{\prime} s^{\prime} t^{\prime}}
$$

But this is easy - the inclusion

$$
D_{r s t} \otimes D_{r^{\prime} s^{\prime} t^{\prime}} \rightarrow W \otimes D_{r s t} \otimes D_{r^{\prime} s^{\prime} t^{\prime}}
$$

induces an isomorphism on $E^{1}$ by the Künneth theorem, so by Proposition 4.3 .

$$
E^{j}\left(W \otimes D_{r s t} \otimes D_{r^{\prime} s^{\prime} t^{\prime}}\right) \cong E^{j}\left(D_{r s t}\right) \otimes E^{j}\left(D_{r^{\prime} s^{\prime} t^{\prime}}\right)
$$

for $j \geq 2$. In particular, $E^{2}\left(W \otimes D_{r s t} \otimes D_{r^{\prime} s^{\prime} t^{\prime}}\right)$ is zero outside of the following set of bidegrees:

$$
\left\{\begin{array}{c}
\left(-s-s^{\prime}, t+t^{\prime}\right) \\
\left(-s-s^{\prime}-r, t+t^{\prime}+r-1\right),\left(-s-s^{\prime}-r^{\prime}, t+t^{\prime}+r^{\prime}-1\right), \\
\left(-s-s^{\prime}-r-r^{\prime}, t+t^{\prime}+r+r^{\prime}-2\right)
\end{array}\right\} .
$$


Proof of Proposition 11.1. Applying $\mathcal{E}$ to the diagram (17), we have the following commutative diagram:

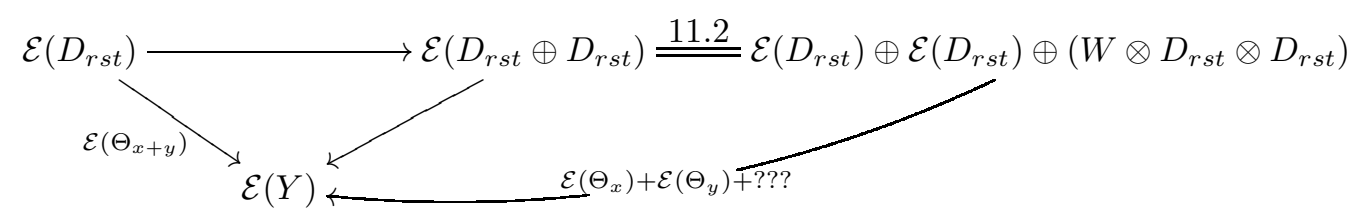

Using the formula from Lemma 11.2 we see that the composite

$$
\mathcal{E}\left(D_{r s t}\right) \rightarrow \mathcal{E}\left(D_{r s t} \oplus D_{r s t}\right) \rightarrow \mathcal{E}\left(D_{r s t}\right) \oplus \mathcal{E}\left(D_{r s t}\right)
$$

is just the diagonal.

We examine the map

$$
E^{2}\left(\mathcal{E}\left(D_{r s t}\right)\right) \rightarrow E^{2}\left(W \otimes D_{r s t} \otimes D_{r s t}\right)
$$

in bidegrees $\{-s\} \times[2 t, \infty)$ and $[-2 s,-s-1] \times\{2 t\}$. Of course $E^{2}\left(W \otimes D_{r s t} \otimes\right.$ $\left.D_{r s t}\right)=E^{2}\left(D_{r s t}\right) \otimes E^{2}\left(D_{r s t}\right)$ is zero at all of these bidegrees except for

$$
E_{-2 s, 2 t}^{2}\left(W \otimes D_{r s t} \otimes D_{r s t}\right)=\mathbb{k} .
$$

In particular, we know that for $m>t-s$

$$
\begin{array}{rr}
\tilde{Q}_{v}^{m}(x+y)=\tilde{Q}_{v}^{m}(x)+\tilde{Q}_{v}^{m}(y) & m \geq t \\
\tilde{Q}_{h}^{m}(x+y)=\tilde{Q}_{h}^{m}(x)+\tilde{Q}_{h}^{m}(y) & m \in(t-s, t]
\end{array}
$$

We saw at the end of the last section that $\tilde{Q}^{t-s}$ is additive.

We will need the following lemma at the beginning of the next section.

Lemma 11.3. We have

$$
E^{2}\left(\mathcal{E}\left(D_{1 s t}\right)\right)=0
$$

which implies

$$
E^{2}\left(\mathcal{E}\left(D_{r s t} \oplus D_{1 s^{\prime} t^{\prime}}\right)\right) \cong E^{2}\left(\mathcal{E}\left(D_{r s t}\right)\right) .
$$

Proof. The implication comes from

$$
\mathcal{E}\left(D_{r s t} \oplus D_{1 s^{\prime} t^{\prime}}\right) \cong \mathcal{E}\left(D_{r s t}\right) \oplus \mathcal{E}\left(D_{1 s^{\prime} t^{\prime}}\right) \oplus\left(W \otimes D_{r s t} \otimes D_{1 s^{\prime} t^{\prime}}\right)
$$

and the fact that $E^{2}\left(W \otimes D_{r s t} \otimes D_{1 s^{\prime} t^{\prime}}\right) \cong E^{2}\left(D_{r s t}\right) \otimes E^{2}\left(D_{1 s^{\prime} t^{\prime}}\right)=0$.

For the main statement, notice that $D_{1 s s}$ is concentrated in homological degree $s$ and $E^{0}=E^{1}$ for this spectral sequence since

$$
H_{k}\left(D_{1 s s}^{p}\right)=\left(D_{1 s s}^{p}\right)_{k}= \begin{cases}\Delta_{s}^{p} & k=s \\ 0 & k \neq s .\end{cases}
$$


The basis for the conormalization of $\mathcal{E}\left(D_{1 s s}\right)$ consists of

$$
\begin{gathered}
e_{n} \otimes \operatorname{id}_{[s]} \otimes \operatorname{id}_{[s]} \\
e_{n} \otimes d^{0} \otimes d^{0}
\end{gathered}
$$

and also

$$
e_{0} \otimes \varepsilon \otimes \varepsilon^{\prime}
$$

where $\varepsilon<\varepsilon^{\prime}$ in $\Delta_{s}^{p}$ and $[1, p] \subset \operatorname{im} \varepsilon \cup \operatorname{im} \varepsilon^{\prime}$. The differential $\delta^{1}$ on a basis element in homological degree $2 s$ is

$$
\delta^{1}\left(e_{0} \otimes \varepsilon \otimes \varepsilon^{\prime}\right)= \begin{cases}e_{0} \otimes d^{0} \varepsilon \otimes d^{0} \varepsilon^{\prime} & \text { when } 0 \in \operatorname{im} \varepsilon \cup \operatorname{im} \varepsilon^{\prime}, \\ 0 & \text { when } 0 \notin \operatorname{im} \varepsilon \cup \operatorname{im} \varepsilon^{\prime} .\end{cases}
$$

We define a contracting homotopy $S$ with

$$
S\left(e_{0} \otimes \varepsilon \otimes \varepsilon^{\prime}\right)= \begin{cases}0 & \text { when } 0 \in \operatorname{im} \varepsilon \cup \operatorname{im} \varepsilon^{\prime}, \\ e_{0} \otimes s^{0} \varepsilon \otimes s^{0} \varepsilon^{\prime}, & \text { when } 0 \notin \operatorname{im} \varepsilon \cup \operatorname{im} \varepsilon^{\prime}\end{cases}
$$

which then satisfies $\delta^{1} S+S \delta^{1}=$ id. For the other degrees, we have

$$
\delta^{1}\left(e_{n} \otimes \operatorname{id}_{[s]} \otimes \operatorname{id}_{[s]}\right)=e_{n} \otimes d^{0} \otimes d^{0}
$$

so we see that $E^{2}=0$.

\section{Definition of the Operations}

In section 10 we defined (additive) operations $\tilde{Q}^{m}$ on $r$-cycles. The goal of this section is to show that these induce operations which are defined on classes in the spectral sequence. The simplest thing would be to show that $\tilde{Q}^{m}$ vanishes on

$$
B_{-s, t}^{r}=\partial Z_{-s+r-1, t-r+2}^{r-1}+Z_{-s-1, t+1}^{r-1}
$$

for all $m$, but this does not happen. It turns out that the horizontal operations $\tilde{Q}_{h}^{m}$ may be nonzero on $\partial Z_{-s+r-1, t-r+2}^{r-1}$, which leads to the indeterminacy in Theorem 12.6 .

We begin with the easy part of $B_{-s, t}^{r}$ : elements in lower filtration.

Lemma 12.1. The homomorphisms $\tilde{Q}^{m}$ vanish on $Z_{-s-1, t+1}^{r-1}$ for $r \geq 2$.

Proof. Write $r^{\prime}=r-1, s^{\prime}=s+1, t^{\prime}=t+1$ and let $y \in Z_{-s^{\prime}, t^{\prime}}^{r^{\prime}}(Y) \subset Z_{s, t}^{r}(Y)$. Then the following commutes

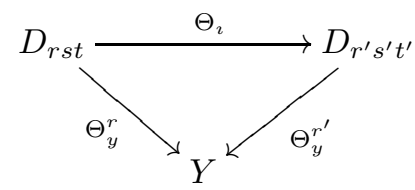


where, of course, we regard $\imath \in Z_{-s^{\prime}, t^{\prime}}^{r^{\prime}}\left(D_{r^{\prime} s^{\prime} t^{\prime}}\right)$ as an element of $Z_{-s, t}^{r}$. If $r^{\prime} \geq 2$, then by Theorem 7.1 the $E^{2}$ page of the spectral sequence of $\mathcal{E}\left(D_{r^{\prime} s^{\prime} t^{\prime}}\right)$ is zero except for the following ranges of bidegrees:

$$
\begin{gathered}
\{-s-1\} \times[2 t+2, \infty) \\
\{-s-r\} \times[2 t+2 r-2, \infty) \\
{[-2 s-2,-s-2] \times\{2 t+2\}} \\
\{-2 s-r-1\} \times\{2 t+r\} \\
{[-2 s-2 r,-s-r-1] \times\{2 t+2 r-2\}}
\end{gathered}
$$

which means that $E^{r}\left(\mathcal{E}\left(D_{r^{\prime} s^{\prime} t^{\prime}}\right)\right)$ is zero on the ranges we are interested in:

$$
\{-s\} \times[2 t, \infty) \text { and }[-2 s,-s-1] \times\{2 t\}
$$

Returning to the definition (15) of $\tilde{Q}$, we note that the diagram

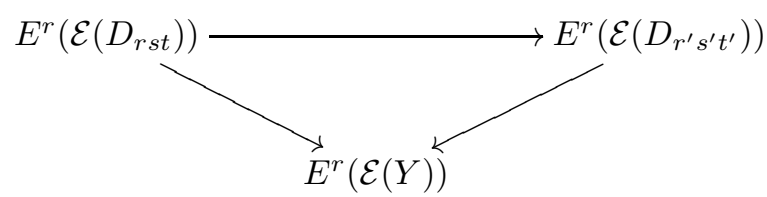

commutes and the rightmost composition takes $u_{p, q}$ to zero for

$$
(p, q) \in\{-s\} \times[2 t, \infty) \cup[-2 s,-s-1] \times\{2 t\},
$$

so all of the $\tilde{Q}$ must vanish on $y$.

The case $r=2$ is even easier, since we know $E^{2}\left(\mathcal{E}\left(D_{1 s^{\prime} t^{\prime}}\right)\right)=0$ by Lemma 11.3

We now shift our attention to $\partial Z_{-s+r-1, t-r+2}^{r-1}$. We run into a problem immediately, for we would like to use the diagram

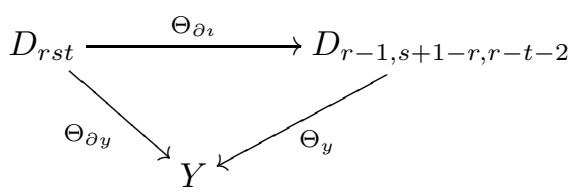

where $y \in Z_{-s+r-1, t-r+2}^{r-1}$, but this diagram does not commute. To see this, write

$$
y=\sum_{j=s-r+1}^{\infty} y^{j} \quad \text { where } \quad y^{j} \in C(Y)^{j} .
$$

We have

$$
\partial y=\sum_{k=s}^{\infty}\left(d_{h} y^{k-1}+d_{v} y^{k}\right) \in F^{-s}
$$


since $y$ is an $(r-1)$-cycle. Then

$$
C\left(\Theta_{\partial y}\right)\left(\sum_{j=s}^{s+r-1} \operatorname{id}_{[j]}\right)=\sum_{j=s}^{s+r-1}\left(d_{h} y^{j-1}+d_{v} y^{j}\right)
$$

and

$$
\begin{aligned}
C\left(\Theta_{y}\right) C\left(\Theta_{\partial \imath}\right)\left(\sum_{j=s}^{s+r-1} \mathrm{id}_{[j]}\right) & =C\left(\Theta_{y}\right)\left(d_{h} \operatorname{id}_{[s-1]}\right) \\
& =d_{h} y^{s-1} .
\end{aligned}
$$

We can guarantee these expressions are equal when $y^{s}, y^{s+1}, \ldots, y^{s+r-1}$ are all zero. We will need the following special case in the proof of Lemma 12.4.

Lemma 12.2. If

$$
y=\sum_{j=s-r+1}^{s-1} y^{j} \in Z_{-s+r-1, t-r+2}^{r-1}
$$

then the diagram

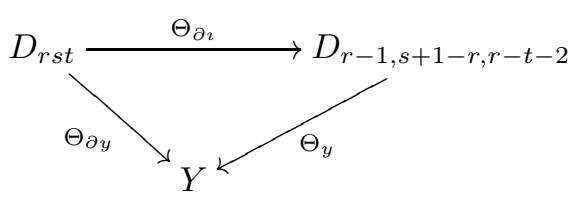

commutes.

Suppose that

$$
y=\sum_{j=s-r+1}^{\infty} y^{j} \in Z_{-s+r-1, t-r+2}^{r-1} .
$$

Then the tail $\sum_{j=s}^{\infty} y^{j}$ is in

$$
F^{-s} \subset Z_{-s+r-1, t-r+2}^{r-1},
$$

so we can split $y$ up into two pieces

$$
y=\sum_{j=s-r+1}^{s-1} y^{j}+\sum_{j=s}^{\infty} y^{j}
$$

both of which are in $Z_{-s+r-1, t-r+2}$. We first treat the tail of $y$.

Proposition 12.3. The homomorphisms $\tilde{Q}^{m}$ vanish on $\partial F^{-s}$. 
Proof. Lemma 12.1guarantees that the operations vanish on elements of $\partial F^{-s-1} \subset$ $Z_{-s-1, t+1}^{r-1}$. By additivity we may consider the boundary of a single element in cosimplicial degree $s$ :

$$
y=y_{t+1}^{s} \text {. }
$$

Define a cosimplicial chain complex $\mathcal{V}$ (depending on $s$ and $t$ ) schematically by

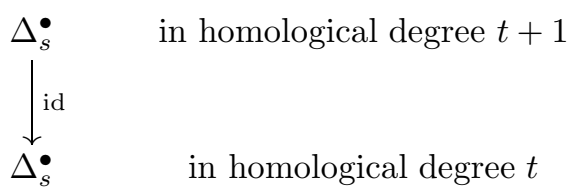

with zero outside of these two homological degrees. The conormalization of this is pictured in the right hand side of Figure 10, as well as a map of bicomplexes from $C\left(D_{r s t}\right)$ to $C(\mathcal{V})$ (open circles map to open circles).

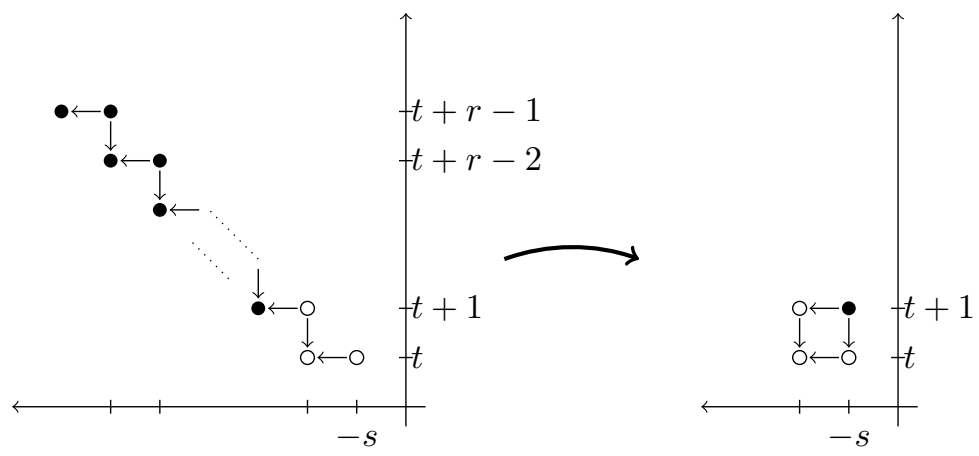

Figure 10: $C\left(D_{r s t}\right) \rightarrow C(\mathcal{V})$

This figure tells us that the diagram

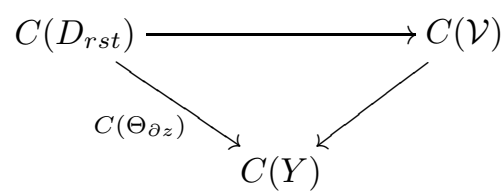

commutes, where $C(\mathcal{V}) \rightarrow C(Y)$ is the map taking the square in the former to the square

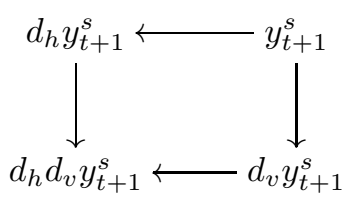


in $C(Y)$. We apply $\mathcal{E}$ to get the commutative diagram

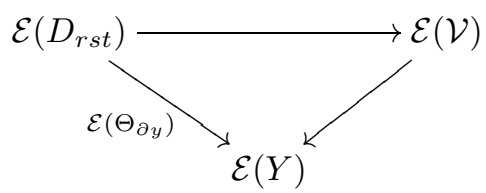

The vanishing of the vertical homology of $\mathcal{V}$ implies the vanishing of $E^{1}(\mathcal{E}(\mathcal{V}))$, so $E^{1}\left(\mathcal{E}\left(\Theta_{\partial y}\right)\right)=0$.

Lemma 12.4. The vertical maps $\tilde{Q}_{v}$ vanish on $\partial Z_{-s+r-1, t-r+2}^{r-1}$ for $r>2$.

Proof. Let $r^{\prime}=r-1, s^{\prime}=s+1-r, t^{\prime}=t-r+2$ and suppose that $y \in$ $Z_{-s+r-1, t-r+2}^{r-1}$ has the form

$$
y=\sum_{j=s-r+1}^{s-1} y^{j}
$$

an assumption that the previous proposition and additivity allow us to make. Since $y$ has this form, the following diagram commutes by Lemma 12.2.

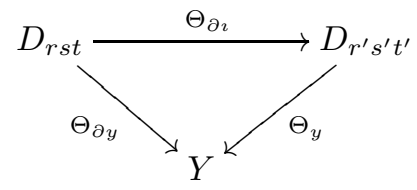

Applying Theorem 7.1 to $\mathcal{E}\left(D_{r^{\prime} s^{\prime} t^{\prime}}\right)$ (when $r>2$ ) we find nonzero terms exactly in the following bidegrees:

$$
\begin{gathered}
\{-s-1-r\} \times[2 t-2 r+4, \infty) \\
\{-s\} \times[2 t, \infty) \\
{[-2 s+2 r-2,-s+r-2] \times\{2 t-2 r+4\}} \\
\{-2 s+2 r-1\} \times\{2 t-r+2\} \\
{[-2 s,-s-1] \times\{2 t\} .}
\end{gathered}
$$

The column $\{-s\} \times[2 t, \infty)$ vanishes at $E^{r}$ by the 'upper left portion' part of Corollary 9.2, so the vertical operations $\tilde{Q}_{v}(\partial y)$ vanish at $E^{r}$.

Lemma 12.5. If $r=2$ then the homomorphisms $\tilde{Q}$ vanish on $\partial Z_{-s+1, t}^{1}$.

Proof. Apply $E^{2} \mathcal{E}$ to the diagram from Lemma 12.2.

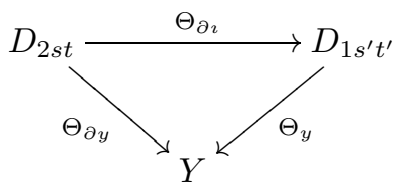

Lemma 11.3 says that $E^{2}\left(\mathcal{E}\left(D_{1 s^{\prime} t^{\prime}}\right)\right)=0$. 
Theorem 12.6. The homomorphisms of Proposition 11.1 induce homomorphisms

$$
\begin{array}{lll}
Q_{v}^{m}: E_{-s, t}^{r}(Y) \rightarrow E_{-s, m+t}^{r}(\mathcal{E}(Y)) & & m \geq t \\
Q_{h}^{m}: E_{-s, t}^{r}(Y) \rightarrow E_{m-s-t, 2 t}^{w}(\mathcal{E}(Y)) & & m \in[t-s, t]
\end{array}
$$

where

$$
w= \begin{cases}r & m=t-s \\ 2 r-2 & m \in[t-s+1, t-r+2] \\ r+t-m & m \in[t-r+3, t] .\end{cases}
$$

Notice that if $r=2$, then $w=2$.

Proof. We have already shown that the vertical operations pass to this quotient using Lemmas 12.1 and 12.4. The well-definedness of the horizontal operations follows from the diagram in Lemma 12.4 by applying the second part of Corollary 9.2 to $D_{r-1, s+1-r, t-r+2}$.

We give an example to show that the $w$ above is the best possible. Consider the diagram

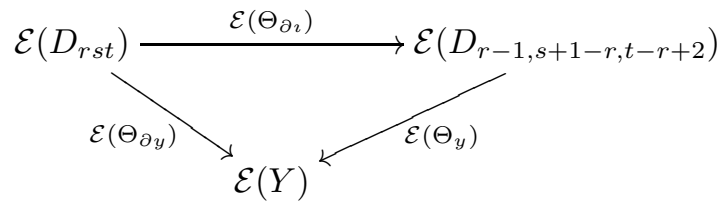

from Lemma 12.4

Example. We let $t=s \geq r-1$, and take as our example the cosimplicial chain complex $Y=D_{r-1, s+1-r, s-r+2}$ together with the class

$$
y=\imath=\sum_{k=s+1-r}^{k} \operatorname{id}_{[k]} .
$$

The diagram

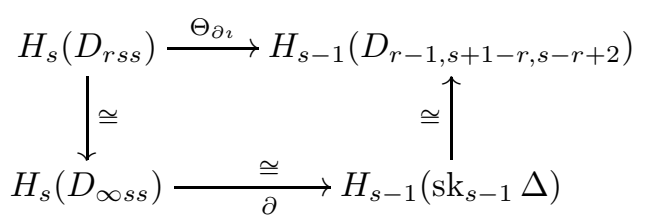

commutes, and so the diagram

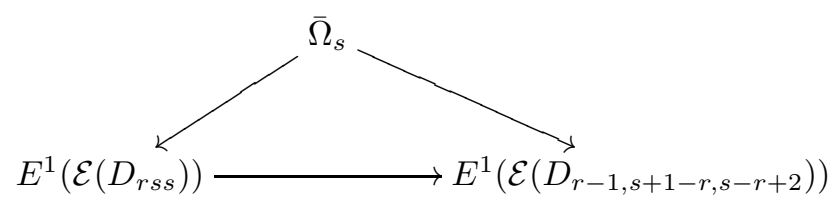


also commutes, where $\bar{\Omega}_{s}$ is the complex defined in (13). Thus, at $E^{2}$, generators in the strip $[-2 s,-s] \times\{2 t\}$ map to nonzero elements. Vanishing of their images occurs at exactly the page described by ' $w$ ' in the theorem.

\section{Acknowledgments}

This work was part of the author's Ph.D. thesis. The author thanks his advisor, Jim McClure, for careful readings and numerous clarifying suggestions. The author would also like to thank Sean Tilson for his interest and for useful discussions about certain parts of this paper. Finally, the many suggestions from the anonymous referee helped the author improve the paper substantially, and for that the author thanks the referee as well.

\section{References}

[1] Anthony P. Bahri. Operations in the second quadrant Eilenberg-Moore spectral sequence. J. Pure Appl. Algebra, 27(3):207-222, 1983.

[2] H.-J. Baues and F. Muro. Cohomologically triangulated categories. II. J. K-Theory, 3(1):1-52, 2009.

[3] J. Michael Boardman. Conditionally convergent spectral sequences. In Homotopy invariant algebraic structures (Baltimore, MD, 1998), volume 239 of Contemp. Math., pages 49-84. Amer. Math. Soc., Providence, RI, 1999.

[4] A. K. Bousfield. On the homology spectral sequence of a cosimplicial space. Amer. J. Math., 109(2):361-394, 1987.

[5] A. K. Bousfield and D. M. Kan. Homotopy limits, completions and localizations. Lecture Notes in Mathematics, Vol. 304. Springer-Verlag, Berlin, 1972.

[6] A. K. Bousfield and D. M. Kan. A second quadrant homotopy spectral sequence. Trans. Amer. Math. Soc., 177:305-318, 1973.

[7] W. G. Dwyer. Higher divided squares in second-quadrant spectral sequences. Trans. Amer. Math. Soc., 260(2):437-447, 1980.

[8] Paul G. Goerss and John F. Jardine. Simplicial Homotopy Theory, volume 174 of Progress in Mathematics. Birkhäuser Verlag, Basel, 1999.

[9] Philip Hackney. Homology operations and cosimplicial iterated loop spaces. Preprint, arXiv:1102.0020 [math.AT].

[10] Philip Hackney. Spectral sequence operations converge to Araki-Kudo operations. Preprint, arXiv:1101.5395 [math.AT]. 
[11] Philip Hackney. Homology Operations in the Spectral Sequence of a Cosimplicial Space. PhD thesis, Purdue University, West Lafayette, Indiana, 2010 .

[12] Daniel M. Kan. Semisimplicial spectra. Illinois J. Math., 7:463-478, 1963.

[13] Tatsuji Kudo and Shôrô Araki. Topology of $H_{n}$-spaces and $H$-squaring operations. Mem. Fac. Sci. Kyūsyū Univ. Ser. A., 10:85-120, 1956.

[14] Hans Ligaard and Ib Madsen. Homology operations in the Eilenberg-Moore spectral sequence. Math. Z., 143:45-54, 1975.

[15] Saunders Mac Lane. Homology. Die Grundlehren der mathematischen Wissenschaften, Bd. 114. Academic Press Inc., Publishers, New York, 1963.

[16] J. Peter May. A general algebraic approach to Steenrod operations. In The Steenrod Algebra and its Applications (Proc. Conf. to Celebrate N. E. Steenrod's Sixtieth Birthday, Battelle Memorial Inst., Columbus, Ohio, 1970), Lecture Notes in Mathematics, Vol. 168, pages 153-231. Springer, Berlin, 1970.

[17] John McCleary. A User's Guide to Spectral Sequences, volume 58 of Cambridge Studies in Advanced Mathematics. Cambridge University Press, Cambridge, second edition, 2001.

[18] Mamoru Mimura and Masamitsu Mori. The squaring operations in the Eilenberg-Moore spectral sequence and the classifying space of an associative $H$-space. I. Publ. Res. Inst. Math. Sci., 13(3):755-776, 1977/78.

[19] David L. Rector. Steenrod operations in the Eilenberg-Moore spectral sequence. Comment. Math. Helv., 45:540-552, 1970.

[20] Brooke E. Shipley. Convergence of the homology spectral sequence of a cosimplicial space. Amer. J. Math., 118(1):179-207, 1996.

[21] William M. Singer. Steenrod Squares in Spectral Sequences, volume 129 of Mathematical Surveys and Monographs. American Mathematical Society, Providence, RI, 2006.

[22] James M. Turner. Operations and spectral sequences. I. Trans. Amer. Math. Soc., 350(9):3815-3835, 1998. 